\title{
A new method for solving the MHD equations in the magnetosheath
}

\author{
C. Nabert $^{1}$, K.-H. Glassmeier ${ }^{1,2}$, and F. Plaschke ${ }^{3}$ \\ ${ }^{1}$ Institut für Geophysik und extraterrestrische Physik, Technische Universität Braunschweig, Germany \\ ${ }^{2}$ Max Planck Institute for Solar System Research, Katlenburg-Lindau, Germany \\ ${ }^{3}$ Institute of Geophysics and Planetary Physics, University of California, Los Angeles, CA, USA \\ Correspondence to: C. Nabert (c.nabert@tu-bs.de)
}

Received: 29 November 2012 - Revised: 13 February 2013 - Accepted: 13 February 2013 - Published: 5 March 2013

\begin{abstract}
We present a new analytical method to derive steady-state magnetohydrodynamic (MHD) solutions of the magnetosheath in different levels of approximation. With this method, we calculate the magnetosheath's density, velocity, and magnetic field distribution as well as its geometry. Thereby, the solution depends on the geomagnetic dipole moment and solar wind conditions only. To simplify the representation, we restrict our model to northward IMF with the solar wind flow along the stagnation streamline. The sheath's geometry, with its boundaries, bow shock and magnetopause, is determined self-consistently. Our model is stationary and time relaxation has not to be considered as in global MHD simulations. Our method uses series expansion to transfer the MHD equations into a new set of ordinary differential equations. The number of equations is related to the level of approximation considered including different physical processes. These equations can be solved numerically; however, an analytical approach for the lowest-order approximation is also presented. This yields explicit expressions, not only for the flow and field variations but also for the magnetosheath thickness, depending on the solar wind parameters. Results are compared to THEMIS data and offer a detailed explanation of, e.g., the pile-up process and the corresponding plasma depletion layer, the bow shock and magnetopause geometry, the magnetosheath thickness, and the flow deceleration.
\end{abstract}

Keywords. Magnetospheric physics (Magnetopause, cusp, and boundary layers; Magnetosheath) - Space plasma physics (Kinetic and MHD theory)

\section{Introduction}

The magnetosheath is the flow region of the solar wind around the Earth. Its characteristics, for example its thickness or magnetic field distribution, depend on the solar wind conditions. In regions without reconnection, the earthward boundary of the flow region, the magnetopause, is defined as a boundary with vanishing normal flow velocity. It is characterized as a small region where a transition from magnetosheath plasma density and temperature to magnetospheric conditions occurs. Very often the magnetopause is identified by an abrupt change in the magnetic field, which is related to a region with spatially confined electric current. Chapman and Ferraro (1930) were the first to speculate about the existence of such a magnetopause current layer. Without a solar wind magnetic field, all current is located at the magnetopause, corresponding to a field-free magnetosheath with a jump of the magnetic field at the magnetopause. Therefore, the flow within the magnetosheath can be treated in terms of hydrodynamics (e.g., Spreiter et al., 1966).

A sharp current layer, however, does not always exist at the magnetopause as demonstrated in Fig. 1, showing observations made on-board the THEMIS-C spacecraft. The five spacecraft of the THEMIS mission were launched in 2007 (Angelopoulos, 2008) and provide a wealth of plasma and magnetic field observations suitable for magnetosheath and magnetopause studies (e.g., Glassmeier et al., 2008; Plaschke et al., 2009; Zhang et al., 2009). On 29 October 2009 the THEMIS-C spacecraft traversed the magnetosheath almost along the stagnation streamline. Measurements by the ESA plasma instrument (McFadden et al., 2008) allow for a clear identification of the magnetopause crossing at 08:25 UT based on abrupt changes of the plasma density and temperature to magnetospheric conditions. Magnetic field 

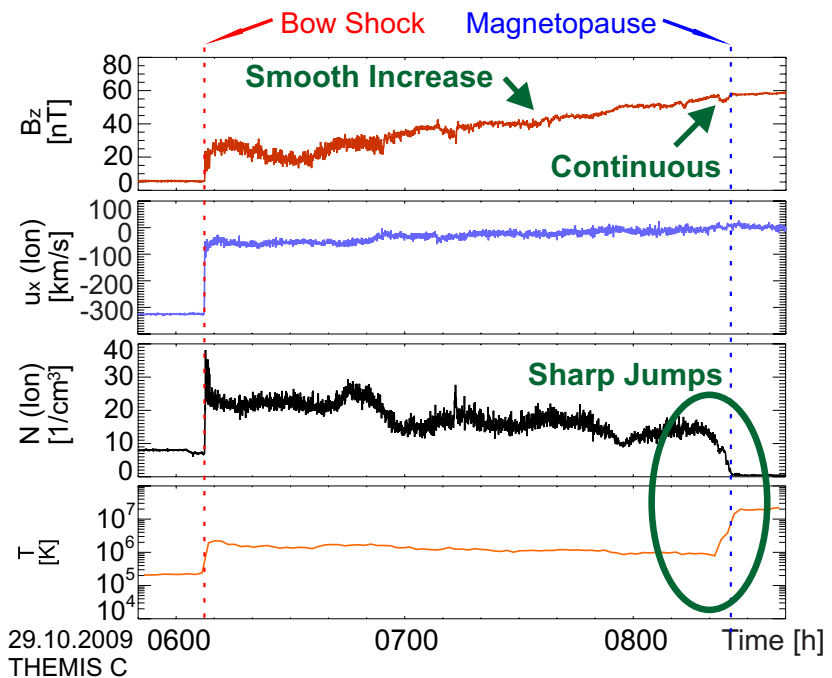

Fig. 1. A low-shear magnetopause transition on 29 October 2009. The upper panel shows $B_{\mathrm{Z}}(\mathrm{GSM})$ magnetic field observations. The second panel displays the (ion) velocity in $\mathrm{x}$-direction (GSM), and the third one shows the density of the plasma ions. The last panel depicts the logarithmic ion temperature.

observations by the FGM (Auster et al., 2008), however, reveal a magnetic pile-up throughout the entire magnetosheath; i.e., the field slowly increases. At the magnetopause, the magnetosheath field smoothly adapts to the magnetospheric field. Thus, there is no confined magnetopause current but currents distributed over the entire magnetosheath. Similar pile-up observations under low-shear conditions were reported by, e.g., Crooker et al. (1979), Paschmann et al. (1993), Phan et al. (1994), or Farrugia et al. (1997). In these earlier reported cases, however, the magnetic pile-up region is usually less extended than in the present case.

A first model to calculate the magnetic pile-up in the magnetosheath was presented by Lees (1964). In this model, the magnetohydrodynamic (MHD) equations are restricted to axisymmetric flows, and the velocity tangential to the stagnation streamline is assumed to be a known function. Although these restrictions strongly limit the scope of the theory, basic aspects of the magnetic pile-up could be investigated. A more detailed theory was developed by Zwan and Wolf (1976), the so-called depletion layer model. They considered a flux tube moving through the magnetosheath in the MHD approach. Zwan and Wolf (1976) concluded that the plasma in the flux tube is squeezed out near the magnetopause, leading to a lowered density and an enhanced magnetic field strength. Note that this consideration leads to a somewhat different density behavior compared to Lees (1964). Another theoretical approach is the magnetic string model by Erkaev et al. (1988). In this model, the MHD equations are solved in a new coordinate system, specially designed to take advantage of the frozen-in magnetic field. However, both latter models also rely on additional assumptions (e.g., the pressure distribution in the magnetosheath) and/or require solutions of more complicated partial differential equations. All these models solve differential equations derived from the MHD equations. Global MHD simulations are another investigation method which solves the complete set of MHD equations directly to obtain a magnetosheath solution as shown by Wu (1984), Wu (1992), Ogino et al. (1992), Siscoe et al. (2002) and Wang et al. (2004), among others.

Simple and very reduced models can provide insight into the basic physical processes underlying a phenomenon. However, more complex models include more processes and can show how the phenomenon is embedded into a complex physical environment. One should be aware, however, that numerical effects can strongly influence any result when solving more complex differential equations. Numerical diffusion near the bow shock or magnetopause is large in global MHD simulations, as noted by Wu et al. (1981). Subsequent developments on the numerical schemes try to reduce the influence of diffusion; however, it is still a difficult task (Toth, 2000). Only a detailed comparison of simple and complex approaches provides a complete understanding of the phenomenon considered.

Here, we present a new analytical method to solve the MHD equations in different orders of approximation, from a lowest-order approach to the complex, full MHD solution. Our approach is able to classify the MHD models introduced above with respect to different levels of approximation. We show that the different results in the density distribution of Lees (1964) and Zwan and Wolf (1976) can be referred back to different levels of approximation used. The method is able to calculate the fluid properties, such as density, pressure, velocity, and magnetic field, as well as the magnetosheath's geometry bounded by the bow shock and magnetopause. In the lowest-order approach, analytical solutions are obtained, yielding explicit expressions for the density and field distribution, as well as for the magnetosheath thickness and its dependence on the solar wind magnetization.

The magnetosheath thickness and the related bow shock distance have been a major topic of investigation for decades, as reviewed by Petrinec (2002). However, only empirical relations have been derived (Spreiter et al., 1966) and modified by Farris and Russell (1994) to take the solar wind magnetic field into account. We will compare our analytically derived expression with the empirical relations.

Finally, our method is applied to two magnetosheath transitions observed by THEMIS: a transition with a confined magnetopause current layer and the transition shown in Fig. 1 exhibiting a strong magnetic pile-up. These observations are compared to MHD magnetosheath solutions in different orders of approximation. 


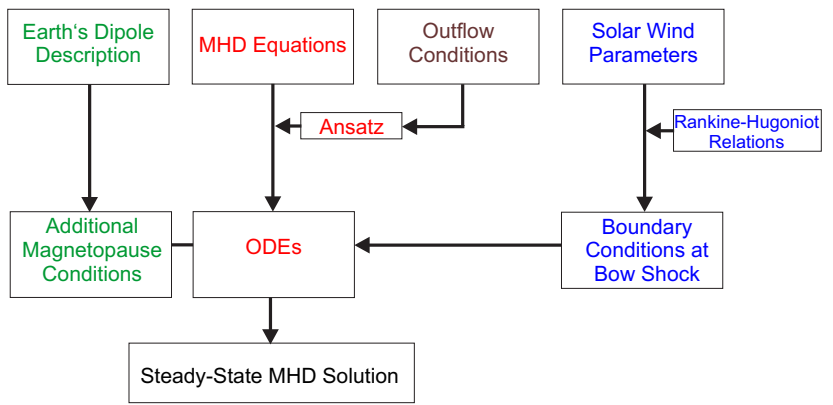

Fig. 2. Overview of the interplay between different aspects of our model. Each color represents a part discussed in a separate section in this chapter. The top row displays the input for our method.

\section{Theory}

Physical quantities in the steady-state magnetosheath, such as density, pressure, velocity, or magnetic field, can be determined by the stationary MHD equations. They depend on three spatial coordinates $(x, y, z)$. We choose the $\mathrm{x}$-axis to be along the stagnation streamline and the $y$ - and $\mathrm{z}$-axis to be perpendicular to it. The quantities are expanded into power series with respect to $y$ and $z$ around the stagnation streamline: $f(x, y, z)=f_{0}(x)+f_{1}(x) y+f_{2}(x) z+f_{3}(x) y z+\ldots$. Substituting such an ansatz for each quantity in the MHD equations and equating coefficients of the tangential orders $(1, y, z, y z, \ldots)$, a system of ordinary differential equations (ODEs) depending only on the normal direction $x$ is obtained. It is suitable to modify our ansatz with respect to the magnetosheath geometry. Consequently, our system of ODEs and the corresponding solutions depend on bow shock and magnetopause geometry parameters. These parameters are calculated by taking the geomagnetic field into account. Boundary conditions (inflow conditions) for our system of ODEs are determined at the bow shock. The shocked values are referred back analytically to the solar wind conditions using the Rankine-Hugoniot relations. Note that the MHD equations require further boundary conditions (in global MHD simulations called outflow conditions) far away along the $y$ - and z-direction. The complete set of ODEs using the series expansion ansatz to infinite order is not easy to handle. A finite expansion order leads to a finite system of ODEs, which remains underdetermined. But we can use the outflow conditions to close the system. Finally, a solution of the magnetosheath flow and field quantities as well as the bow shock and magnetopause geometry can be calculated depending on solar wind conditions only. The interplay of the different parts of the model are displayed in Fig. 2.

\subsection{Deriving ODEs from MHD}

Plenty of phenomena of the plasma flow in the magnetosheath are described by the ideal MHD theory (e.g., Siscoe et al., 2002). The time-independent (stationary) MHD equa-

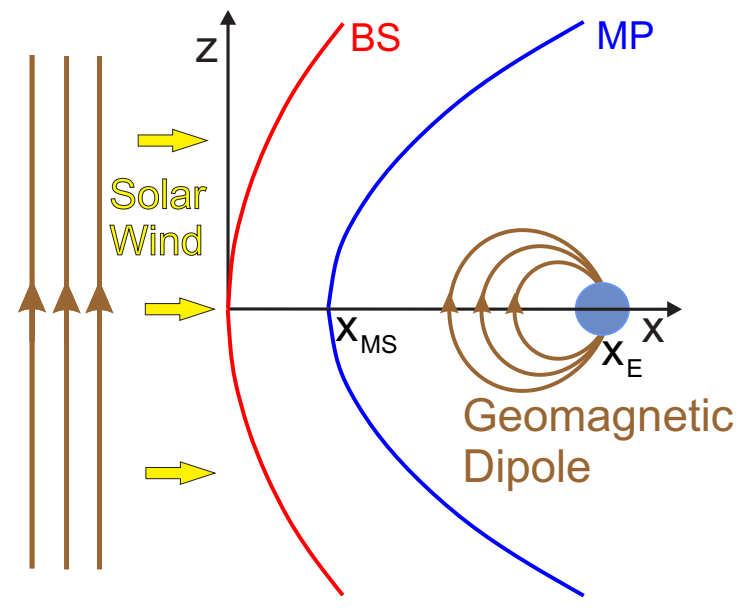

Fig. 3. The incident solar wind in $x$-direction with its field along the $z$-direction on the left side and the Earth with its dipole field on the right side. The origin of the Cartesian coordinate system $(x, y, z)$ shall be at the nose of the bow shock (BS).

tions read

$\nabla \cdot(\rho \boldsymbol{u})=0$,

$\rho(\boldsymbol{u} \cdot \nabla) \boldsymbol{u}+\nabla p-\frac{1}{\mu_{0}}(\nabla \times \boldsymbol{B}) \times \boldsymbol{B}=0$,

$\nabla \times(\boldsymbol{u} \times \boldsymbol{B})=0$

$\nabla \cdot \boldsymbol{B}=0$

$p=k \rho^{\gamma}$.

Here $\rho$ denotes mass density, $\boldsymbol{u}$ the fluid's bulk velocity, $p$ the thermal gas pressure (assumed to be isotropic), $\boldsymbol{B}$ the magnetic field, $\mu_{0}=4 \pi \times 10^{-7} \mathrm{~N} / \mathrm{A}^{2}$ the vacuum permeability, and $\gamma$ the ratio of specific heats. Note that instead of the full energy conservation law, an adiabatic law (Eq. 5) is used with the proportionality constant $k$. The adiabatic law is valid within the magnetosheath, but not through the bow shock due to entropy changes.

The super-magnetosonic solar wind flow causes a bow shock in front of the Earth. A Cartesian coordinate system is used with origin at the bow shock's nose (subsolar point). The $\mathrm{x}$-axis points towards the Earth, which is located at $x_{\mathrm{E}}$ (see Fig. 3). The bow shock distance to the Earth, that is, $x_{\mathrm{E}}$, will be determined later as part of the solution. We choose the incident solar wind to flow along the $\mathrm{x}$-axis. The $\mathrm{z}$-axis is normal to the ecliptic pointing northward, and the y-axis completes the right-hand triad. For simplification, we choose the 
solar wind magnetic field to be parallel (northward or positive) along the z-axis. Furthermore, the Earth's magnetic field is represented by a dipole, its moment being anti-parallel to the $\mathrm{z}$-axis with magnitude $M=8 \times 10^{15} \mathrm{Tm}^{3}$. The components of the geomagnetic field $\boldsymbol{B}_{\mathrm{E}}$ are given by (e.g., Ogino, 1993)

$B_{\mathrm{E}, \mathrm{x}}=\frac{-3 z \Delta x}{r^{5}} M$

$B_{\mathrm{E}, \mathrm{y}}=\frac{-3 y z}{r^{5}} M$

$B_{\mathrm{E}, \mathrm{z}}=\frac{-2 z^{2}+\Delta x^{2}+y^{2}}{r^{5}} M$,

with the radial distance $r=\sqrt{\Delta x^{2}+y^{2}+z^{2}}$, and $\Delta x=$ $x_{\mathrm{E}}-x$ defining the distance from the Earth's center (Fig. 3). Note that the solar wind magnetic field and the dipole field are parallel close to the dayside stagnation streamline, which excludes reconnection.

The situation introduced above is highly symmetric. The dipole field has a rotational symmetry with respect to the dipole moment axis, and the solar wind flow is perpendicular to this symmetry axis, leading to symmetric properties in the MHD flow around the dipole field. A detailed discussion of the symmetry relations is presented in Appendix A.

We approximate the bow shock as well as the magnetopause shapes by elliptic paraboloids (functional relation $x=x_{0}+a y^{2}+b z^{2}$ with constants $x_{0}, a$, and $b$ ). This corresponds to a second-order series expansion in accordance with the symmetry considerations. The bow shock and magnetopause parametrization are

$x=\sum_{t=y, z} c_{\mathrm{BS}, t} t^{2}$,

$x=x_{\mathrm{MS}}+\sum_{t=y, z} c_{\mathrm{MP}, t} t^{2}$,

respectively, where $x_{\mathrm{MS}}$ denotes the magnetopause distance from the bow shock's nose (which is the magnetosheath thickness along the stagnation streamline, $\mathrm{x}$-axis). The constant parameters $c_{\mathrm{BS}, t}$ for the bow shock and $c_{\mathrm{MP}, t}$ for the magnetopause represent curvatures in the respective tangential direction $t=y$ or $t=z$. These geometric parameters are determined by the interaction of the magnetosheath plasma with the dipole field as we will show later.

The flow and field variables in the magnetosheath are expanded into Taylor series with respect to the $y$ - and $z-$ direction around the stagnation streamline $(y=z=0)$. Due to the symmetry relations summarized in Table A1 (Appendix A), several expansion terms vanish. This leads to the following ansatz expanded to the third order:

$\rho=\rho_{0}+\rho_{20} y^{2}+\rho_{02} z^{2}$ $p=p_{0}+p_{20} y^{2}+p_{02} z^{2}$,

$B_{\mathrm{x}}=B_{\mathrm{x} 01} z+B_{\mathrm{x} 21} y^{2} z+B_{\mathrm{x} 03} z^{3}$,

$B_{\mathrm{y}}=B_{\mathrm{y} 11} y z$

$B_{\mathrm{z}}=B_{\mathrm{z} 0}+B_{\mathrm{z} 20} y^{2}+B_{\mathrm{z} 02} z^{2}$,

$u_{\mathrm{x}}=u_{\mathrm{x} 0}+u_{\mathrm{x} 20} y^{2}+u_{\mathrm{x} 02} z^{2}$

$u_{\mathrm{y}}=u_{\mathrm{y} 10} y+u_{\mathrm{y} 12} y z^{2}+u_{\mathrm{y} 30} y^{3}$

$u_{\mathrm{z}}=u_{\mathrm{z} 01} z+u_{\mathrm{z} 21} y^{2} z+u_{\mathrm{z} 03} z^{3}$.

Note that the expansion only holds for the tangential directions. Thus, the coefficients are arbitrary functions of $x$. Their indices indicate the order in $y$ and $z$; e.g., " 20 " means second order in $y$ and zeroth order in $z$ and " 0 " means zeroth order in $y$ and $z$.

Due to the curved shape of the bow shock and magnetopause, coefficient functions are more conveniently depending on $\tilde{x}$ instead of $x$ (e.g., $\left.\rho_{0}(x) \rightarrow \rho_{0}(\tilde{x})\right)$, with $\tilde{x}$ defined by the following relation:

$x=\tilde{x}+\sum_{t=y, z}\left(c_{\mathrm{BS}, t}+\Delta c_{t} \frac{\tilde{x}}{x_{\mathrm{MS}}}\right) t^{2}$,

where $\Delta c_{t}=c_{\mathrm{MP}, t}-c_{\mathrm{BS}, t}$ denotes the difference between the magnetopause curvature and the bow shock curvature. Each value for $\tilde{x}$ corresponds to an elliptic paraboloid defined by Eq. (19). In particular, $\tilde{x}=0$ corresponds to the bow shock parametrization (Eq. 9) and $\tilde{x}=x_{\mathrm{MS}}$ to the magnetopause parametrization (Eq. 10). Therefore, the modified ansatz describes the physical quantities on elliptic paraboloids parametrized by $\tilde{x}$, starting at the bow shock for $\tilde{x}=0$ and finishing at the magnetopause at $\tilde{x}=x_{\mathrm{MS}}$ (see Fig. 4). Note that the modification of the ansatz does not affect its symmetry because $\tilde{x}$ depends on $y^{2}$ and $z^{2}$ only and, thus, $\tilde{x}(y, z)$ is independent of the sign of $y$ and $z$. This can be seen solving Eq. (19) with respect to $\tilde{x}$. Thus, the ansatz still satisfies the symmetry relations given in Table A1.

Substituting our ansatz into the MHD system (1)-(5) yields a set of ordinary differential equations (ODEs) by equating the coefficients of zeroth order,

$\left(\rho_{0} u_{\mathrm{x} 0}\right)^{\prime}+\rho_{0}\left(u_{\mathrm{y} 10}+u_{\mathrm{z} 01}\right)=0$, 


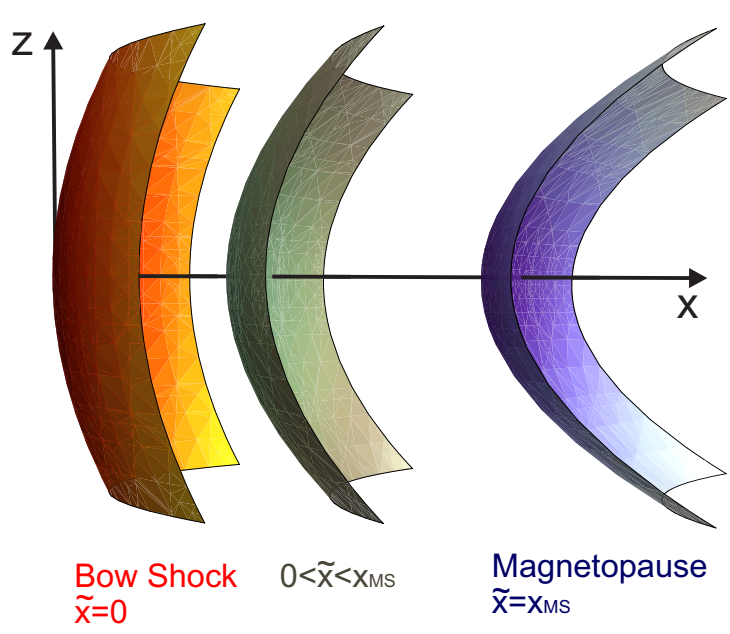

Fig. 4. Three-dimensional sketch of the elliptic paraboloids described by Eq. (19) for different $\tilde{x}$. The bow shock on the left side for $\tilde{x}=0$ and the magnetopause on the right side for $\tilde{x}=x_{\mathrm{MS}}$.

$\left(B_{\mathrm{z} 0} u_{\mathrm{x} 0}\right)^{\prime}+B_{\mathrm{z} 0} u_{\mathrm{y} 10}=0$,

$\rho_{0} u_{\mathrm{x} 0} u_{\mathrm{x} 0}^{\prime}+p_{0}^{\prime}+B_{\mathrm{z} 0} B_{\mathrm{z} 0}^{\prime}-B_{\mathrm{x} 01} B_{\mathrm{z} 0}=0$,

$p_{0}=k \rho_{0}^{\gamma}$,

and first order,

$$
B_{\mathrm{x} 01}^{\prime}+2 B_{\mathrm{z} 02}+B_{\mathrm{y} 11}-2 \tilde{c}_{\mathrm{z}} B_{\mathrm{z} 0}^{\prime}=0
$$

$$
\rho_{0} u_{\mathrm{x} 0} u_{\mathrm{y} 10}^{\prime}+\rho_{0} u_{\mathrm{y} 10}^{2}+2\left(p_{20}+B_{\mathrm{z} 0} B_{\mathrm{z} 20}\right)-B_{\mathrm{y} 11} B_{\mathrm{z} 0}
$$$$
-2 \tilde{c}_{\mathrm{y}}\left(p_{0}^{\prime}+B_{\mathrm{z} 0} B_{\mathrm{z} 0}^{\prime}\right)=0,
$$

$$
\rho_{0} u_{\mathrm{x} 0} u_{\mathrm{z} 01}^{\prime}+\rho_{0} u_{\mathrm{z} 01}^{2}+2 p_{02}+B_{\mathrm{x} 01}^{2}-B_{\mathrm{x} 01} B_{\mathrm{z} 0}^{\prime}
$$$$
-2 \tilde{c}_{\mathrm{Z}} p_{0}^{\prime}=0 \text {, }
$$

$$
p_{20}=k \gamma \rho_{20} \rho_{0}^{\gamma-1},
$$

$$
\begin{array}{r}
p_{02}=k \gamma \rho_{02} \rho_{0}^{\gamma-1}, \\
B_{\mathrm{y} 11} u_{\mathrm{x} 0}+2\left(B_{\mathrm{z} 02} u_{\mathrm{x} 0}+B_{\mathrm{z} 0} u_{\mathrm{x} 02}\right) \\
-B_{\mathrm{x} 01}\left(u_{\mathrm{y} 10}+2 u_{\mathrm{z} 01}\right)+2 \tilde{c}_{\mathrm{z}} B_{\mathrm{z} 0} u_{\mathrm{y} 10}=0,
\end{array}
$$

with $\tilde{c}_{t}=c_{\mathrm{BS}, t}+\Delta c_{t} \frac{\tilde{x}}{x_{\mathrm{MS}}}$. Derivatives with respect to $\tilde{x}$ are marked with a prime. To simplify the reading of the equations, we set $\mu_{0}=1$. Equations obtained from equating coefficients of the second order can be found in Appendix B. We expressed the series expansion of the MHD quantities in new coordinates $\tilde{x}, y, z$. However, as the MHD equations are explicitly expressed in Cartesian coordinates, the chain rule was applied to compute the derivatives with respect to $\tilde{x}$, e.g., $\partial_{x} p=p^{\prime} \partial_{\mathrm{x}} \tilde{x}$. The factor $\partial_{\mathrm{x}} \tilde{x}$ can be calculated from Eq. (19):

$$
\partial_{\mathrm{x}} \tilde{x}=1-\sum_{t=y, z} \frac{\Delta c_{t}}{x_{\mathrm{MS}}} t^{2}+O\left(t^{4}\right) .
$$

Here $t^{4}$ denotes any fourth-order product of $y$ and $z$. The system of equations presented contains a conservation equation (Eq. 29), which has been satisfied by boundary conditions only, as it does not contain any derivatives. We denote Eqs. (20)-(23) as the zeroth-order system, Eqs. (20)-(28) as the first-order system, and Eqs. (20)-(28) and (B1)-(B7) as the second-order system.

Our ansatz transforms the stationary MHD equations, which are partial differential equations (PDEs), into a set of ordinary differential equations: (20)-(28) and (B1)-(B7). Although the number of equations increases, the numerical effort to solve ordinary differential equations (ODEs) is significantly lower. Hence, for a sufficient order the solution might be obtained with less effort. The second-order system presented here contains 16 ODEs, depending on 22 coefficient functions and 5 geometric parameters. Thus, 6 additional equations are required to calculate all coefficient functions, and 5 additional conditions are needed to determine the geometric parameters.

\subsection{Solar wind at the bow shock boundary}

First-order derivatives of the coefficient functions with respect to $\tilde{x}$ in the above ODEs require knowledge of corresponding boundary values at one point $\tilde{x}_{0}=0$, i.e., $\rho_{0}\left(\tilde{x}_{0}\right)$, $\rho_{20}\left(\tilde{x}_{0}\right), \rho_{02}\left(\tilde{x}_{0}\right), \ldots$ We choose to set these values at $\tilde{x}_{0}=0$ (bow shock) using shocked solar wind parameters. These are related to the solar wind via Rankine-Hugoniot relations (e.g., Petrinec and Russell, 1997):

$\left[\rho u_{\xi}\right]=0$,

$\left[B_{\xi}\right]=0$,

$\left[\rho u_{\xi}^{2}+p+\frac{\boldsymbol{B}_{\tau}^{2}}{2 \mu_{0}}\right]=0$,

$\left[\rho u_{\xi} \boldsymbol{u}_{\tau}-\frac{B_{\xi}}{\mu_{0}} \boldsymbol{B}_{\tau}\right]=0$, 
$\left[u_{\xi} \boldsymbol{B}_{\tau}-\boldsymbol{u}_{\tau} B_{\xi}\right]=0$,

$$
\left[\left(\frac{\rho}{2} \boldsymbol{u}^{2}+\left(\frac{\gamma}{\gamma-1}\right) p+\frac{\boldsymbol{B}_{\tau}^{2}}{\mu_{0}}\right) u_{\xi}-\frac{B_{\xi}}{\mu_{0}} \boldsymbol{B}_{\tau} \boldsymbol{u}_{\tau}\right]=0 .
$$

The squared brackets $[\ldots]$ indicate that the quantity therein is conserved across the shock. The subscript $\xi$ denotes the normal component and $\tau$ tangential components with respect to the bow shock. Solving the Rankine-Hugoniot relations with respect to the bow shock geometry (Eq. 9) and the chosen solar wind conditions, the shocked values are obtained. Power series expansion in $\mathrm{y}$ - and $\mathrm{z}$-direction of this solution and equating coefficients with our ansatz determine the coefficient functions of the ansatz at $\tilde{x}=0$. Analytical solutions for the zeroth-order coefficients can be found in, e.g., Siscoe (1983). A brief summary of his work and a detailed analytical approach for the the higher-order coefficient values up to the second order are given in Appendix C.

As a result, the velocity coefficients are given by

$u_{l i j}(\tilde{x}=0)=f_{u}(i, j) 2^{i+j} c_{B S, y}^{i} c_{B S, z}^{j} \Delta u$,

where $\Delta u=u_{\mathrm{SW}}-u_{\mathrm{x} 0}(\tilde{x}=0), l \in\{x, y, z\}, i$ and $j$ are labeled as in Eqs. (16)-(18), and $f_{u}(i, j)$ is defined by (signfunction)

$f_{u}(i, j)=\left\{\begin{array}{ll}+1, & i+j \in\{1\} \\ -1, & i+j \in\{2,3\}\end{array}\right.$.

The magnetic field coefficients are given by:

$B_{l i j}(\tilde{x}=0)=f_{B}(i, j) 2^{i+j} c_{\mathrm{BS}, y}^{i} c_{\mathrm{BS}, z}^{j} \Delta B$,

in which

$f_{B}(i, j)=\left\{\begin{array}{ll}+1, & i+j \in\{1,2\} \\ -1, & i+j \in\{3\}\end{array}\right.$,

where $\Delta B=B_{\mathrm{SW}}-B_{\mathrm{z} 0}(\tilde{x}=0), l \in\{x, y, z\}$, and $i$ and $j$ are as in Eqs. (13)-(15). The boundary values for the density and pressure coefficients $\rho_{20}, \rho_{02}, p_{20}$, and $p_{02}$ are approximately zero.

\subsection{Outflow boundary conditions}

Since the MHD system (1)-(5) contains partial derivatives with respect to all three space dimensions, boundary conditions are needed for three linearly independent planes. In the previous section, boundary conditions were defined for the bow shock plane; these are commonly called inflow conditions in global MHD simulations. Here the remaining set of outflow boundary conditions are defined, used to close the system of ODEs.
The higher-order coefficient functions in the series expansion dominate far away from the stagnation streamline (e.g., $\left.\rho(\tilde{x}, y \rightarrow \infty, z \rightarrow \infty) \approx \rho_{20}(\tilde{x}) y^{2}+\rho_{02}(\tilde{x}) z^{2}\right)$. We suggest setting the highest-order coefficients to their bow shock boundary values. This condition is similar to common outflow boundary condition in simulations (e.g., Ogino, 1993). Different, specific choices of explicit functional relations to close the system of ODEs lead basically to the models by Lees (1964) and Zwan and Wolf (1976), as discussed below.

\subsection{Conditions from the geomagnetic field as an obstacle}

Despite the boundary conditions introduced above, the system of ODEs still contains five undetermined geometric parameters: the bow shock curvatures $c_{\mathrm{BS}, \mathrm{y}}$ and $c_{\mathrm{BS}, \mathrm{z}}$, the magnetopause curvatures $c_{\mathrm{MP}, \mathrm{y}}$ and $c_{\mathrm{MP}, \mathrm{z}}$, and the magnetosheath thickness $x_{\text {MS }}$. To determine these, inner boundary conditions are necessary.

First, the flow should be tangential with respect to the magnetopause, not allowing flow to penetrate into the magnetosphere (e.g., Biernat et al., 1999). This stems from the definition of the magnetopause as earthward flow boundary. The flow direction at the magnetopause can be calculated using magnetopause surface coordinates. The normal magnetopause vector is derived from the magnetopause parametrization (10):

$\xi_{\mathrm{MP}}=\frac{1}{n_{\mathrm{MP}}}\left(1,-2 c_{\mathrm{MP}, \mathrm{y}} y,-2 c_{\mathrm{MP}, \mathrm{z}} z\right)^{T}$,

where $n_{\mathrm{MP}}=\sqrt{1+\sum_{t=y, z} 4 c_{\mathrm{MP}, t}^{2} t^{2}}$ normalizes the vector. A vanishing normal flow velocity through the magnetopause yields $\boldsymbol{\xi}_{\mathrm{MP}} \cdot \boldsymbol{u}\left(\tilde{x}=x_{\mathrm{MS}}, y, z\right)=0$. This expression holds for the $y$ - and z-direction, so using the velocity ansatz (16)-(18) yields

$u_{\mathrm{x} 0}\left(\tilde{x}=x_{\mathrm{MS}}\right)=0$,

$c_{\mathrm{MP}, \mathrm{y}}=\frac{u_{\mathrm{x} 20}\left(\tilde{x}=x_{\mathrm{MS}}\right)}{2 u_{\mathrm{y} 10}\left(\tilde{x}=x_{\mathrm{MS}}\right)}$,

$c_{\mathrm{MP}, \mathrm{z}}=\frac{u_{\mathrm{x} 02}\left(\tilde{x}=x_{\mathrm{MS}}\right)}{2 u_{\mathrm{z} 01}\left(\tilde{x}=x_{\mathrm{MS}}\right)}$.

However, at the magnetopause surface the flow velocity vanishes along the magnetic field direction $\left(\rightarrow u_{\mathrm{z} 01}\left(\tilde{x}=x_{\mathrm{MS}}\right)=\right.$ 0 ) as pointed out by Siscoe et al. (2002). Thus, in the presence of magnetic fields Eq. (44) is no longer valid. Instead, we use the assumption that magnetic fields of different origin cannot mix (Frozen-in theorem). This yields the vanishing of any normal magnetic field component at the magnetopause boundary: $\boldsymbol{\xi}_{\mathrm{MP}} \cdot \boldsymbol{B}\left(\tilde{x}=x_{\mathrm{MS}}, y, z\right)=0$ (e.g., Biernat 
et al., 1999). This requires

$c_{\mathrm{MP}, \mathrm{z}}=\frac{B_{\mathrm{x} 01}\left(\tilde{x}=x_{\mathrm{MS}}\right)}{2 B_{\mathrm{z} 0}\left(\tilde{x}=x_{\mathrm{MS}}\right)}$.

Secondly, a restricting condition arises from the Earth's dipole field itself. The total magnetic field of our MHD solution $(\boldsymbol{B})$ should by a superposition of the magnetic fields generated by magnetosheath currents $\left(\boldsymbol{B}_{j}\right)$ and the Earth's magnetic field $\left(\boldsymbol{B}_{\mathrm{E}}\right)$. Since the Earth's dipole field is curlfree (see Eqs. 6-8), the curl of the total field yields the current distribution in the magnetosheath $\boldsymbol{j}_{\mathrm{MS}}$ only:

$\nabla \times \boldsymbol{B}=\nabla \times \boldsymbol{B}_{j}=\mu_{0} \boldsymbol{j}_{\mathrm{MS}}$.

The magnetic field generated by these currents can be calculated with Biot-Savart's law. The superposition of this magnetosheath-current-field and the Earth's magnetic field necessarily has to match the total field from our MHD solution. If this is not satisfied, geometric parameters and the magnetopause distance to the Earth's center have to be modified accordingly.

A more simple approach to take the geomagnetic field into account uses a pressure condition at the magnetopause which is valid in the hydrodynamic limit. Mead and Beard (1964) pointed out that the tangential components of the total magnetic field determine the (calculated) total pressure behind the magnetopause $p_{\text {tot,MP: }}$

$p_{\mathrm{tot}, \mathrm{MP}}=\frac{\left(\boldsymbol{\xi}_{\mathrm{MP}} \times\left(\boldsymbol{B}_{\mathrm{E}}+\boldsymbol{B}_{\boldsymbol{j}}\right)\right)^{2}}{2 \mu_{0}}$.

With respect to Mead and Beard (1964), a good first approximation for the right-hand side yields:

$p_{\text {tot,MP }}=\frac{\left(f \boldsymbol{B}_{\mathrm{E}} \times \boldsymbol{\xi}_{\mathrm{MP}}\right)^{2}}{2 \mu_{0}}$,

with $f=2.44$. This expression is valid near the stagnation point and determines the magnetopause distance to the Earth's center. Variations in y- and z-direction give additional conditions for determination of two curvature parameters.

Hence, the conditions of vanishing normal flow and field component and the condition for the Earth's magnetic field determine the geometric parameters $x_{\mathrm{MS}}, c_{\mathrm{MP}, \mathrm{y}}, c_{\mathrm{MP}, \mathrm{z}}, c_{\mathrm{BS}, \mathrm{y}}$ and $c_{\mathrm{BS}, \mathrm{z}}$. The differential equations together with the closure conditions for the highest-order coefficients and the inner boundary conditions determine a unique solution for given solar wind parameters.

\subsection{Application of the method}

To calculate magnetosheath solutions with our method, first an appropriate order of approximation has to be chosen. For example, the second-order model gives a good approximation of the dayside magnetosheath up to several $R_{\mathrm{E}}$ beside the stagnation streamline. If the solar wind is along the stagnation streamline (i.e., the $\mathrm{x}$-direction) with a perpendicular, northward IMF, we can directly apply the ODEs presented here. The system of ODEs related to the zeroth order is given by Eqs. (20)-(23). The second-order approach is given by Eqs. (20)-(28) and (B1)-(B7) as presented in Sect. 2.1 and Appendix B. For solar wind conditions violating the required symmetry conditions, ansatz (11)-(18) has to be replaced by an arbitrary series expansion. However, the derivation of the corresponding ODEs is analogous. Further, higher-order approximations require higher-order series expansions. We set the coefficient functions of the highest-order constant at their postshock values to close the system of equations (i.e., for the second-order approach presented, $u_{\mathrm{y} 30}, u_{\mathrm{y} 12}, u_{\mathrm{z} 03}, u_{\mathrm{z} 21}$, $B_{\mathrm{x} 30}$, and $B_{\mathrm{x} 21}$ are constant). The boundary conditions for the ODEs $(\rho(\tilde{x}=0), p(\tilde{x}=0), \boldsymbol{u}(\tilde{x}=0)$, and $\boldsymbol{B}(\tilde{x}=0))$ are determined at the bow shock by solving the Rankine-Hugoniot relations for the solar wind conditions $\left(\rho_{\mathrm{SW}}, p_{\mathrm{SW}}, \boldsymbol{u}_{\mathrm{SW}}\right.$, and $\left.\boldsymbol{B}_{\mathrm{SW}}\right)$ with respect to the shock geometry. However, we can also use the analytical approach presented in Appendix C yielding equations for zeroth-order coefficients (Eqs. C1C4) and for the higher-order coefficients (Eqs. C19 and C27). The second-order terms of density and pressure vanish in this approach.

The Rankine-Hugoniot relations as well as the ODEs require knowledge of the geometric parameters $x_{\mathrm{MS}}, c_{\mathrm{MP}, \mathrm{y}}$, $c_{\mathrm{MP}, \mathrm{z}}, c_{\mathrm{BS}, \mathrm{y}}$, and $c_{\mathrm{BS}, \mathrm{z}}$. As an initial choice, we can use the analytical expressions (D13), (D24), (D28), (D25), and (D29) with the magnetopause distance (Eq. D23). They are related to solar wind conditions via Eqs. $(\mathrm{C} 1)-(\mathrm{C} 4)$. The solution of our system has to satisfy the inner boundary conditions given by Eqs. (42), (43), (45), and (48). The latter condition holds for the $y$ - and z-direction. The geometric parameters need to be determined self-consistently; i.e., the initial choice has to be modified until the conditions are satisfied. Note that higher-order expansions of the bow shock and magnetopause curvatures contain more parameters and lead to more inner boundary conditions. The zeroth- and first-order systems are not able to determine the geometric parameters self-consistently.

Each system of equations together with the boundary and closure conditions represents a distinct approach to obtain a magnetosheath solution, characterized by the order of the system and corresponding level of approximation.

\section{Relations to other models}

Our zeroth-order equations provide a model similar to Lees (1964). Extending Lees's first considerations, we present an analytical approach for the solution of the equations, by which the physical quantities (density, pressure, magnetic field, and velocity) and the magnetosheath geometry (bow shock, magnetopause, and magnetosheath thickness) are obtained as a function of solar wind conditions only. The 
complete derivation is presented in Appendix D. In the following section, we focus on an important result of this calculation: the analytical expression for the magnetosheath thickness. Furthermore, we show that the models by Lees (1964), Zwan and Wolf (1976), and Erkaev et al. (1996) are included in our new method for different orders of approximation.

\subsection{Analytical expression for the magnetosheath thickness}

As presented in Appendix D, an analytical expression of the magnetosheath thickness can be found in the zeroth-order approximation that is solely dependent on solar wind conditions. This expression is given by

$x_{\mathrm{MS}}=\frac{\Delta x_{\mathrm{MP}}}{\left(\frac{4}{5}+m_{\mathrm{BS}}\right)\left(g_{u}-1\right)-1}$,

where $g_{u}=u_{\mathrm{SW}} / u_{\mathrm{x} 0}(0)$, the subsolar velocity jump at the bow shock, which is introduced in Appendix C (Eq. C3) as a function of solar wind conditions. Furthermore, the magnetopause distance to the Earth's center $\Delta x_{\mathrm{MP}}$ is given as a function of solar wind conditions in Eq. (D23), and $m_{\mathrm{BS}}$ is a measure for the solar wind magnetization given by

$m_{\mathrm{BS}}=1-\frac{1}{1+\frac{\gamma}{2} \frac{p_{0}(0)}{p_{\mathrm{mag}}(0)}}$.

Here $p_{\text {mag }}(0)=B_{\mathrm{z} 0}^{2}(0) / 2 \mu_{0}$ is the postshock magnetic pressure and $\gamma=5 / 3$ is the ratio of specific heats. The magnetosheath thickness is proportional to the magnetopause distance to the Earth's center. A typical value for the magnetopause distance is about $10 R_{\mathrm{E}}$; therewith, the magnetosheath thickness results in $2.3 R_{\mathrm{E}}$ in the hydrodynamic limit $\left(m_{\mathrm{BS}}=1\right)$ for high Mach numbers $\left(g_{u}=4\right)$. The magnetosheath thickness increases with solar wind magnetic field. It should be noted that the sheath thickness may become negative in Eq. (49). This occurs for very low Mach number conditions, for which conditions our approximation is not valid. A very often used empirical relation based on hydrodynamic considerations (Spreiter et al., 1966) is

$x_{\mathrm{MS}}=1.1 \frac{1}{g_{u}} \Delta x_{\mathrm{MP}}$.

This equation offers the same proportionality between magnetosheath thickness and magnetopause distance. Farris and Russell (1994) modified this relation and obtained

$x_{\mathrm{MS}}=1.1 \frac{(\gamma-1) M_{1, \mathrm{SW}}^{2}+2}{(\gamma+1)\left(M_{1, \mathrm{SW}}^{2}-1\right)} \Delta x_{\mathrm{MP}}$,

where $M_{1, \mathrm{SW}}$ is the solar wind magnetosonic Mach number (see also, Bennett et al., 1997). Figure 5 shows a comparison between this relation and Eq. (49). The upper panel shows the magnetosheath thickness as a function of the unmagnetized
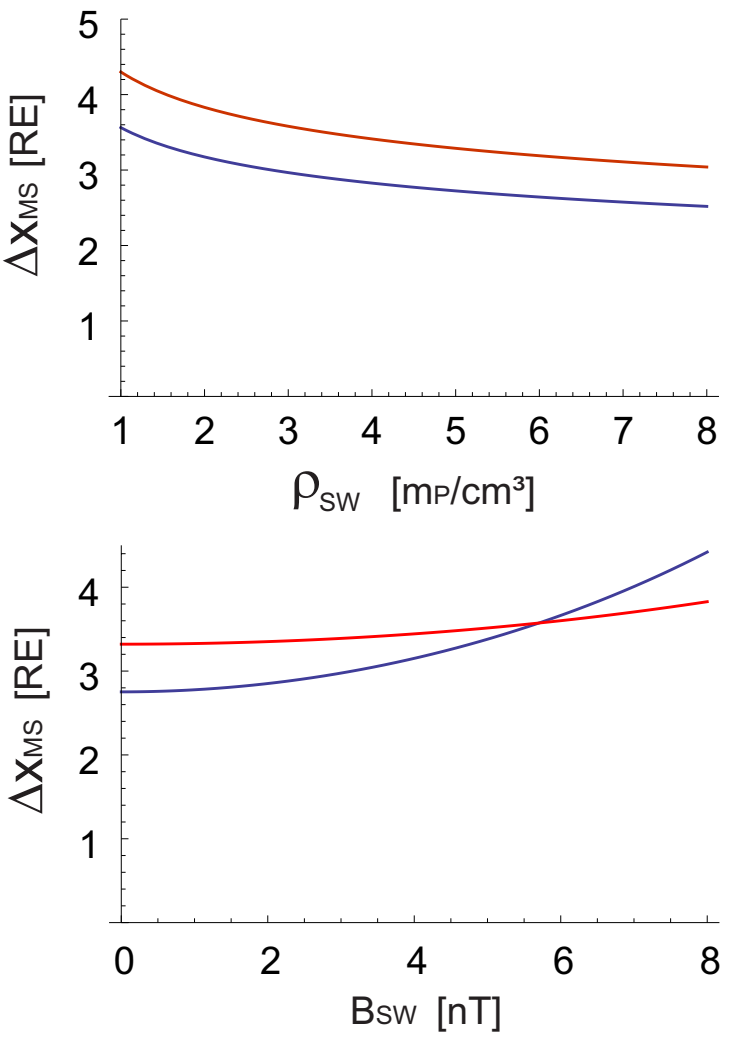

Fig. 5. Magnetosheath thickness of our model by Eq. (49) (blue curves) in comparison to the model by Farris and Russell (1994) (red curves). The upper plot shows the density dependence of the sheath thickness for $u_{\mathrm{SW}}=400 \mathrm{~km} \mathrm{~s}^{-1}, T_{\mathrm{SW}}=2 \times 10^{5} \mathrm{~K}$, and $B_{\mathrm{SW}}=0 \mathrm{nT}$. The lower panel offers the magnetic field dependence for $u_{\mathrm{SW}}=400 \mathrm{~km} \mathrm{~s}^{-1}, T_{\mathrm{SW}}=2 \times 10^{5} \mathrm{~K}$, and $\rho_{\mathrm{SW}}=8.4 \times$ $10^{-21} \mathrm{~kg} \mathrm{~m}^{-3}=5 \mathrm{~m}_{\mathrm{P} \mathrm{cm}^{-3}}$.

solar wind's density. The relations are similar; however, an offset of about $20 \%$ is observed. In the lower panel, both relations' dependence on solar wind magnetic field are shown. Eq. (49) yields a steeper increase in the sheath's thickness. However, for tyical solar wind conditions both relations provide a similar magnetosheath thickness, in accord with observations. Thus, Eq. (49) is a suitable analytical expression for the magnetosheath thickness as a function of solar wind conditions.

\subsection{The Lees approach}

Lees (1964) presented a first model considering the effects of a solar wind magnetic field on the magnetosheath on the stagnation streamline. Starting from ideal MHD and considering the same solar wind conditions, he derives equations similar to our zeroth-order differential equations (20)(23). Note that these equations do not depend on the bow shock and magnetopause geometry. Neglecting the magnetic shear $\left(\rightarrow B_{\mathrm{x} 01} B_{\mathrm{z} 0}=0\right)$ and assuming an axisymmetric flow (i.e., $u_{\mathrm{y} 10}=u_{\mathrm{z} 01}$ ) in our zeroth-order system leads to Lees's 
equations. As noted, a relation to close the system is required. He suggested determining the divergence function $u_{\mathrm{y} 10}$ as part of the solution along the magnetopause away from the stagnation streamline without going into details. Lees's model is a zeroth-order approach to the flow problem; we extended his considerations by calculating analytical solutions presented in Appendix D. As shown in our approach, higher-order differential equations allow for a self-consistent determination of the divergence functions influencing the solution.

\subsection{The depletion model}

The depletion model by Zwan and Wolf (1976) investigates, in more detail, density and magnetic field variations on the stagnation streamline and also in its vicinity. Starting from the time-dependent ideal MHD equations, they derive a system of two-dimensional partial differential equations describing the properties of a magnetic flux tube moving through the magnetosheath. Approximations such as WBK and Taylor expansion restrict the calculations to the vicinity of the stagnation streamline. Although the model by Zwan and Wolf (1976) depends on several limiting assumptions, the picture of the pile-up process was deepened. They conclude that during northward IMF plasma is squeezed out of flux tubes close to the magnetopause, inducing a density decrease corresponding to a magnetic field increase in order to maintain pressure balance. The region of density decrease, called the depletion region, is narrower than predicted by Lees's simple model. In the model of Zwan and Wolf (1976), initial postshock divergence values also need to be determined, similarly to our model. However, they obtain these values from numerical hydrodynamic calculations after Spreiter et al. (1966). To close their system, the functional relation of the pressure is assumed to match the hydrodynamic simulations, too. If we applied the same pressure condition to our model, we would achieve closure of our first-order system (20)-(28). This suggests a correspondence of our first-order system with the model of Zwan and Wolf (1976) despite the approaches in solving the MHD system of equations being rather different (we solve only ODEs). Note that the secondorder velocity coefficient functions of our model do not contribute to the first-order equations and thus do not need to be determined.

\subsection{The magnetic string approach}

The magnetic string approach by Erkaev et al. (1988) transfers the stationary ideal MHD equations into a different set of partial differential equations (PDEs), using so-called material coordinates (e.g., Erkaev et al., 2003). These coordinates are similar to frozen-in coordinates, with directions along the flow velocity, the magnetic field, and the electric field (Pudovkin and Semenov, 1977b). These coordinates are preferably used in ideal MHD due to the frozen-in theorem.
The MHD equations can be simplified assuming the total pressure given in the entire magnetosheath, which leads to a set of two-dimensional PDEs that describes thin magnetic flux tubes. Similar to our method, a parametrized bow shock is used. The bow shock and the magnetopause curvatures are calculated self-consistently using magnetopause boundary conditions. These conditions are a vanishing normal velocity and magnetic field component, and they assume that the pressure satisfies the Newtonian approximation (Petrinec and Russell, 1997). The magnetic string approach was used to investigate the magnetic barrier region, which is equivalent to the depletion layer region. It was applied to the magnetosheath region of Earth (Farrugia et al., 1997), Venus (Biernat et al., 1999), and Jupiter (Erkaev et al., 1996). Further considerations with respect to anisotropic pressure are presented by Erkaev et al. (2000).

\section{Application to THEMIS observations}

We apply our method to two different scenarios: The hydrodynamic magnetosheath transition - i.e., the solar wind magnetic field is zero - and the magnetic pile-up transition as shown in Fig. 1, where the solar wind is significantly magnetized.

\subsection{Hydrodynamic transition}

Without a solar wind magnetic field, we expect a field-free magnetosheath and results comparable to the hydrodynamic calculations by, e.g., Spreiter et al. (1966). We choose the solar wind velocity to be $u_{\mathrm{SW}}=310 \mathrm{~km} \mathrm{~s}^{-1}$, the solar wind density $\rho_{\mathrm{SW}}=1.34 \times 10^{-20} \mathrm{~kg} \mathrm{~m}^{-3}$, the solar wind magnetic field $B_{\mathrm{SW}}=0.02 \mathrm{nT}$, and the solar wind temperature $T_{\mathrm{SW}}=1.75 \times 10^{5} \mathrm{~K}$. These conditions agree with actual solar wind conditions during THEMIS C's crossing of the magnetosheath region on 24 August 2008. The probe traversed the magnetosheath about $5.5 R_{\mathrm{E}}$ in y-direction and about $2 R_{\mathrm{E}}$ in z-direction away from the stagnation streamline $\left(R_{\mathrm{E}}=6371 \mathrm{~km}\right)$. Solar wind measurements were obtained by THEMIS B, which was closely following THEMIS C.

We apply our method to this event using the analytical (zeroth-order) approach presented in Appendix D, the full zeroth-order approach with the equations solved numerically, and the second-order approach. The results of the calculations and the THEMIS C observations on 24 August 2008 are displayed in Fig. 6. The numerical zeroth-order approach yields the smaller magnetosheath thickness. The analytical and second-order approach (solutions on the stagnation streamline) yield comparable thicknesses. The observed magnetosheath thickness, however, is about $0.4 R_{\mathrm{E}}$ larger. This difference is explained by the distance of the probe's orbit to the stagnation streamline, reflected in better agreement of the solution of the second-order system on the probe's orbit (black) to observations as shown in Fig. 6 . 


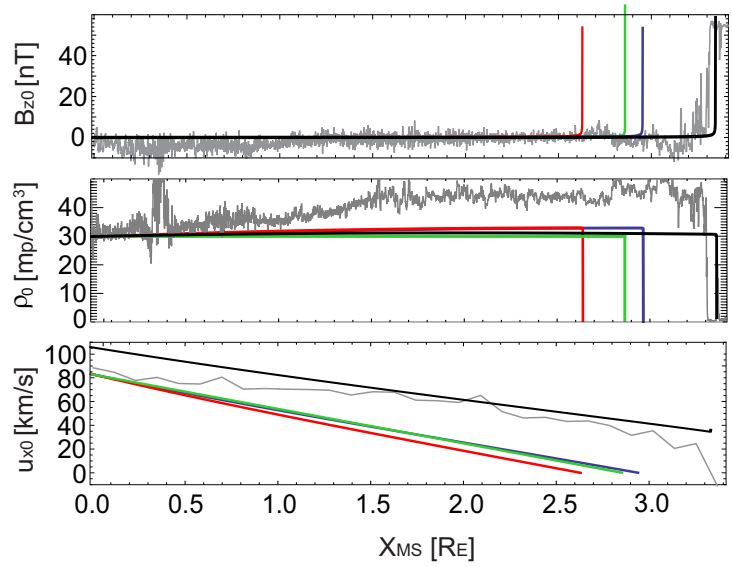

Fig. 6. Magnetosheath solutions on the stagnation streamline for the analytical (green), the numerical zeroth order (red), and the second order approach (blue). The second-order solution on the probe's orbit (close to but not exactly on the stagnation streamline) is shown in black, the corresponding THEMIS data of 24 August 2008 in grey.

The analytical magnetic field diverges at the magnetopause, due to the linear approximation of the velocity's xcomponent. A slight density increase is apparent in all solutions but the analytical one. This results from the conversion of dynamic pressure, neglected only in the analytical calculations, into gas pressure. However, the measured density increases at a higher rate than that given by the models, which is attributed to a density increase in the solar wind density of about $15 \%$ during the observation time. All calculated velocities decrease nearly linearly. On the stagnation streamline the velocity's x-component vanishes, but close to it a finite value remains, vanishing after the magnetopause. This behavior is in accord with the measured data.

The second-order approach reveals the velocity distribution within the three-dimensional magnetosheath geometry. The corresponding streamlines in the xy-plane are displayed in Fig. 7. The density distribution in the xy-plane is shown in Fig. 8, which shows a decreasing density away from the stagnation streamline. Although these density variations appear to be small, corresponding pressure gradients affect the solution significantly.

The results presented are in agreement with the THEMIS observations as well as numerical calculations by Spreiter et al. (1966) over a large part of the dayside magnetosheath. Our three-dimensional solution of the second-order approach with self-consistently computed bow shock and magnetopause geometries yields results that best match the observations of all models considered.

\subsection{Northward IMF transition}

Without magnetic reconnection, the IMF piles up in the magnetosheath against the magnetopause. THEMIS C observations on 29 October 2009 (Fig. 1), showing such a pile-up,

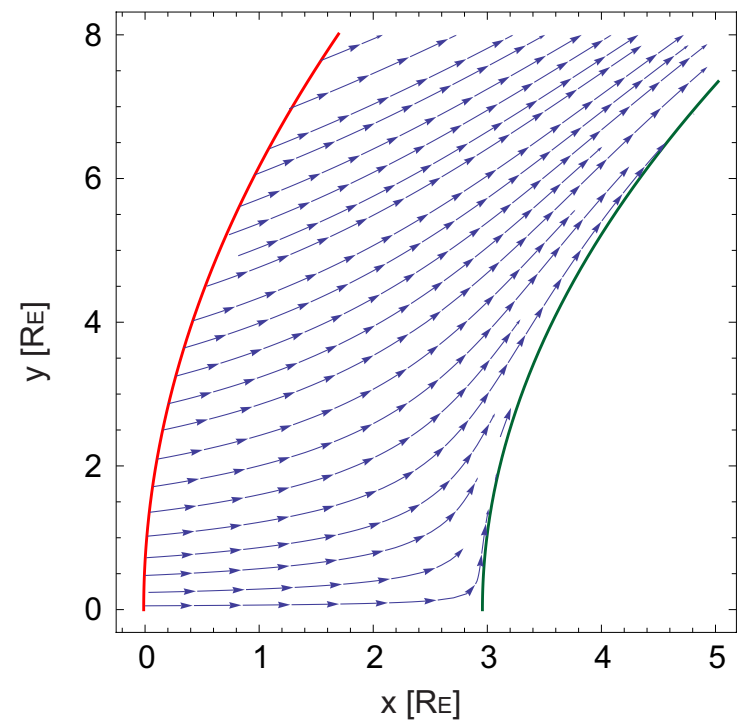

Fig. 7. Magnetosheath streamlines with the velocity obtained by solving the second-order approach. The parabolic bow shock (red) and the magnetopause (green) are delineated.

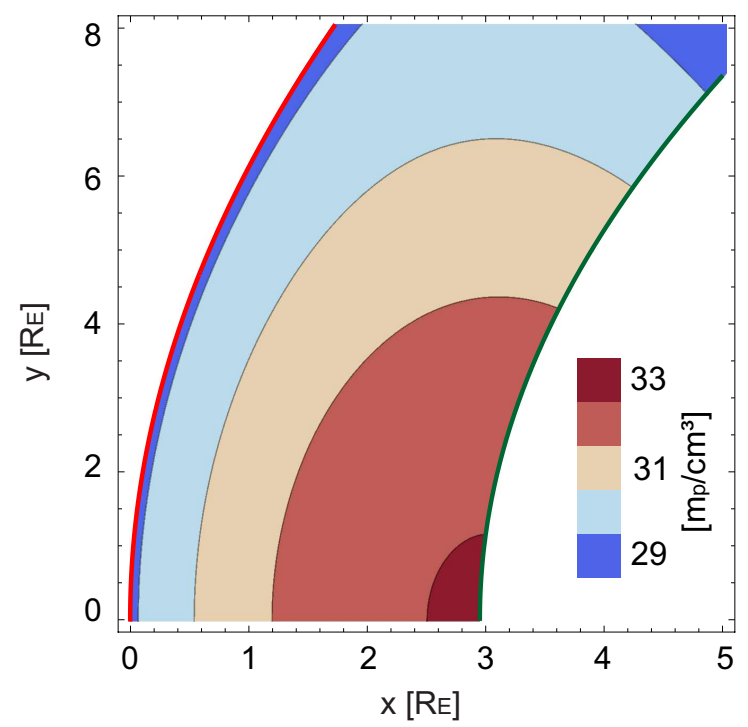

Fig. 8. Magnetosheath density distribution obtained by solving the second-order approach. The parabolic bow shock (red) and the magnetopause (green) are delineated.

are compared to results of our method using different orders of approximation. The data shown are just a part of the complete magnetosheath observation. About $1.5 \mathrm{~h}$ before the bow shock crossing shown in the figure, two further bow shock crossings were registered by the THEMIS C spacecraft. During the whole time interval, the solar wind velocity was about $325 \mathrm{~km} \mathrm{~s}^{-1}$, the temperature $2.1 \times 10^{5} \mathrm{~K}$, and the solar wind magnetic field between $5.0 \mathrm{nT}$ and $5.5 \mathrm{nT}$. Note that the magnetic field is purely northward with minor deviations of less than $45^{\circ}$ (in the last part of the transition even less than $10^{\circ}$ ) 


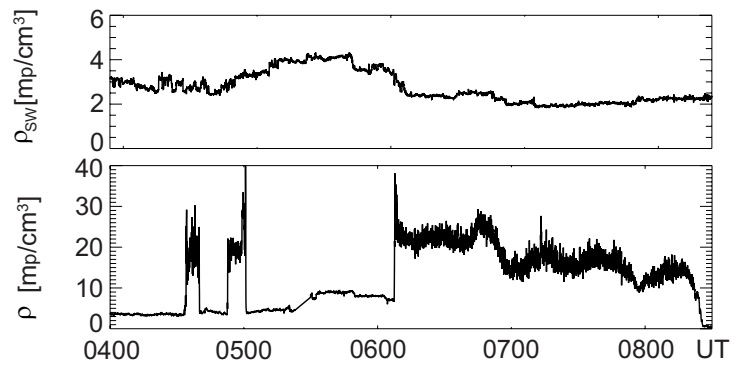

Fig. 9. The solar wind density measured by THEMIS B and the density measured by THEMIS $\mathrm{C}$ crossing the bow shock several times. The absolute values of the solar wind density do not fit well. This might be explained by a non-vanishing spacecraft potential; however, the relative variations can be considered. Note that the density jump measured by THEMIS $\mathrm{C}$ at about 05:30 UT is related to a mode change of the ESA plasma instrument of the spacecraft (McFadden et al., 2008).

and the satellite's trajectory is located close to the stagnation streamline (distance less than $2 R_{\mathrm{E}}$ ) as required for our method in the approximations presented here. However, the solar wind density varied significantly during the observation time, which violates the assumed stationarity of our model with the consequences discussed below. Time-shifted solar wind (ion) density measurements (observed by THEMIS B) and corresponding THEMIS C density measurements, both obtained by the on-board ESA plasma instrument (McFadden et al., 2008), are shown in Fig. 9. Immediately after the first two bow shock crossings at 04:30 UT and 04:50 UT, the solar wind density increases, causing an inward motion of bow shock and magnetopause. Consequently, the THEMIS C spacecraft reenters the solar wind at about 05:00 UT.

The bow shock distance to the Earth's center and that of the magnetosheath thickness of the second-order solution are shown in Fig. 10. Both quantities decrease with increasing density. This is also consistent with the results of our analytical approach (Eqs. 49 and D23). Additionally, Fig. 10 shows that the effect of the magnetic field variability during the event discussed is negligible when compared to changes in density.

As can be seen in Fig. 9, a solar wind density decrease preceded the bow shock crossing of THEMIS C at 06:05 UT. This causes an outward motion of the bow shock. Therefore, the velocity behind the bow shock shown in Fig. 1 is about $20 \mathrm{~km} \mathrm{~s}^{-1}$ lower than expected by the Rankine-Hugoniot relations (Eqs. 31-36), which are only valid for a stationary situation. Consequently, the bow shock and magnetopause position and the magnetosheath thickness varied during the first part of the observation. For this reason, the length scales of the observations might not be comparable to our calculations. The results of our analytical approach, our zeroth-order as well as our second-order approach are presented in Fig. 11.

In all cases, a magnetic pile-up is predicted. As explained by Siscoe et al. (2002), the magnetosheath plasma at the mag-
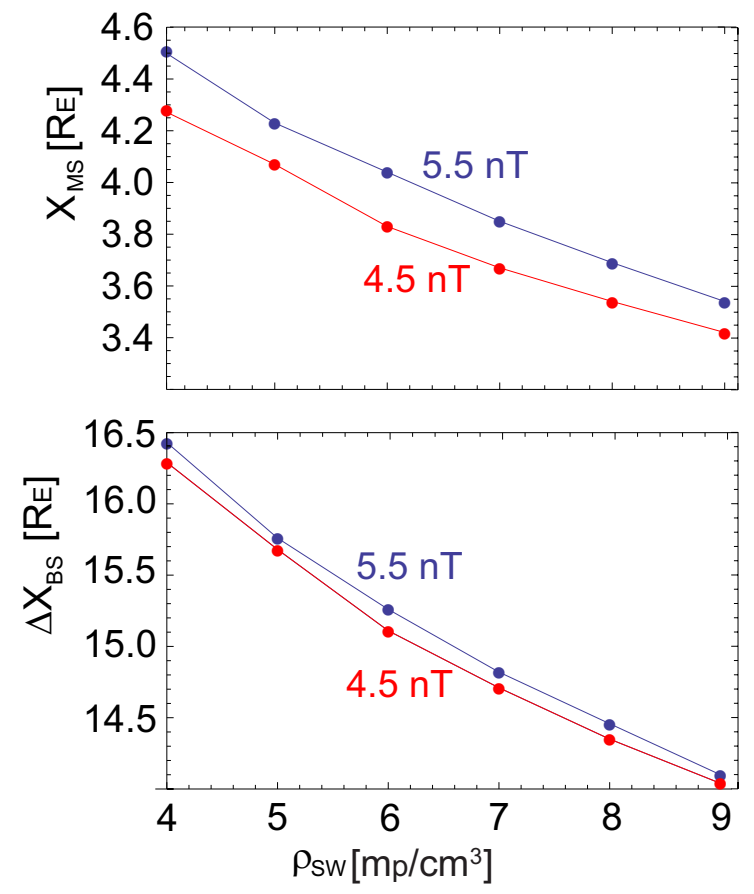

Fig. 10. Calculations of the bow shock distance from the Earth's center and the magnetopause thickness as a function of the solar wind density, using the second-order approach. Two different solar wind magnetizations, $4.5 \mathrm{nT}$ and $5.5 \mathrm{nT}$, were considered.

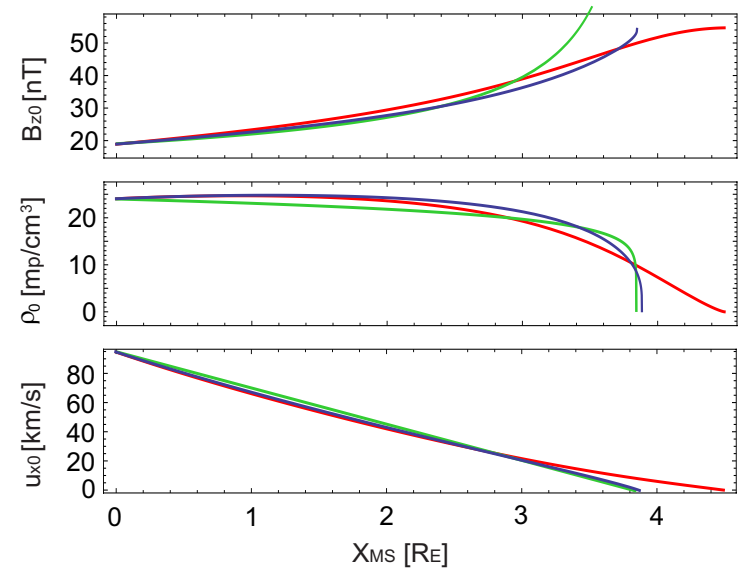

Fig. 11. Magnetosheath solutions on the stagnation streamline for the analytical (green), the numerical zeroth-order (red), and the second-order approach (blue).

netopause surface can flow in two dimensions at the surface, whereas the magnetic field can only escape in one direction, because field lines are already one-dimensional. Therefore, more plasma escapes than magnetic field in front of the magnetopause, leading to a pile-up of the magnetic field. Note that the pile-up region exists in the hydrodynamic case above, too; however, the pile-up region is located closely to the magnetopause. 
Again, the magnetic field of the analytical approach diverges at the magnetopause due to the linear velocity approximation made. Furthermore, the numerical zeroth-order approach shows the most-extended sheath thickness. This behavior is associated with a slow density decrease, which contrasts with the density drop at the magnetopause in the other calculations. The solution of the zeroth-order system is comparable to that of the Lees (1964) model, which exhibits the same slow density decrease, whereas the second-order solution is similar to the solution by Zwan and Wolf (1976). The pressure gradients in the second-order approach enhance the plasma flow away from the stagnation streamline close to the magnetopause. Consequently, the depletion layer thickness, which is given by the density decrease at the magnetopause, differs.

It can be shown that the often-used empirical magnetopause model by Shue et al. (1998) and the bow shock model by Bennett et al. (1997) provide approximately the same curvatures as our theoretical approach. Furthermore, the second-order approach offers a well-known behavior: The divergence of the velocity along the magnetic field drops at the magnetopause. This is related to the fact that the stagnation point is extended to a line along the magnetic field at the magnetopause as discussed by Pudovkin and Semenov (1977a).

\section{Conclusions}

A method to calculate the properties of the magnetosheath as part of the solar wind-geomagnetic field interaction by solving the stationary MHD equations for different levels of approximation is presented. The solutions are calculated without considering time relaxation into a stationary state, as required, for instance, by MHD simulations. A power series ansatz is introduced which transfers the stationary MHD equations into a set of ordinary differential equations (ODEs). The number of equations is determined by the order of approximation considered in the ansatz. In the process, for simplicity, we restricted the approach to northward IMF and a solar wind along the stagnation streamline. The postshock values, used as boundary conditions for the ODEs, are referred back to solar wind conditions via Rankine-Hugoniot relations. Furthermore, typical outflow boundary conditions are chosen to determine a unique solution. The Earth's dipole field yields additional restrictions for the solution determining the magnetosheath's geometry.

A full analytical solution of our zeroth-order approach is presented, which results in an analytical expression of the magnetosheath thickness (Eq. 49). The models by Lees (1964), Zwan and Wolf (1976), and Erkaev et al. (1988) are classified by different orders of approximation with respect to our method, revealing similarities and differences and providing a more detailed insight into magnetosheath phenomena.
Table A1. Symmetries of the situation considered. Axisymmetry of a quantity $f(x, y, z)$ to the xy-plane means $f(x, y,-z)=$ $f(x, y, z)$, point symmetry means $f(x, y,-z)=-f(x, y, z)$, analogous with respect to the xz-plane.

\begin{tabular}{ccc}
\hline Phys. quantity & Symmetry to $x y$ & Symmetry to $x z$ \\
\hline$B_{\mathrm{X}}$ & axisym. & pointsym. \\
$B_{\mathrm{y}}$ & pointsym. & pointsym. \\
$B_{\mathrm{Z}}$ & axisym. & axisym. \\
$u_{\mathrm{X}}$ & axisym. & axisym. \\
$u_{\mathrm{y}}$ & pointsym. & axisym. \\
$u_{\mathrm{Z}}$ & axisym. & pointsym. \\
$\rho$ & axisym. & axisym. \\
$p$ & axisym. & axisym. \\
\hline
\end{tabular}

We apply our method to THEMIS observations. First, a hydrodynamic transition is examined featuring the typical Chapman-Ferraro picture of a jump in the magnetic field at the magnetopause. The second transition investigated shows an extended magnetic pile-up region resulting from a significantly magnetized solar wind. Model results in terms of streamline distribution, the density contours, the magnetic field behavior, and the magnetosheath geometry validate our approach.

We are currently exploring the possibility to generalize our approach to situations at other planetary bodies such as comets (Glassmeier et al., 2007) or asteroids (Auster et al., 2010).

\section{Appendix A}

\section{Symmetry relations}

The symmetry relations of the MHD variables with respect to the $\mathrm{xy}$ - and xz-coordinate planes are summarized in Table A1.

In the following, we sketch the derivation of these relations. In Sect. 2.1, we assume the solar wind to flow along the $\mathrm{x}$-axis with its magnetic field along the z-axis and the Earth's dipole field given by Eqs. (6)-(8). Both the undisturbed solar wind and the dipole field satisfy the symmetry relations. The time evolution of the flow is governed by the time-dependent MHD equations, which read (e.g., Wu, 1992)

$\dot{\rho}+\nabla \cdot(\rho \boldsymbol{u})=0$,

$\rho \dot{\boldsymbol{u}}+\rho(\boldsymbol{u} \cdot \nabla) \boldsymbol{u}+\nabla p-\frac{1}{\mu_{0}}(\nabla \times \boldsymbol{B}) \times \boldsymbol{B}=0$,

$\dot{B}-\nabla \times(\boldsymbol{u} \times \boldsymbol{B})=0$, 
$\nabla \cdot \boldsymbol{B}=0$

$\dot{\epsilon}+\nabla \cdot\left(\left(\frac{\rho \boldsymbol{u}^{2}}{2}+\frac{\gamma}{\gamma-1} p\right) \boldsymbol{u}-\frac{1}{\mu_{0}}(\boldsymbol{u} \times \boldsymbol{B}) \times \boldsymbol{B}\right)=0$.

Here $\rho$ denotes mass density, $\boldsymbol{u}$ the fluid's bulk velocity, $p$ the thermal gas pressure assumed to be isotropic, $\boldsymbol{B}$ the magnetic field, $\mu_{0}=4 \pi \times 10^{-7} \mathrm{~N} / \mathrm{A}^{2}$ the vacuum permeability, and $\epsilon=\rho \boldsymbol{u}^{2} / 2+\boldsymbol{B}^{2} /\left(2 \mu_{0}\right)+p /(\gamma-1)$ the energy density with $\gamma$ as the ratio of specific heats. Time derivatives are presented in dot notation. The time-dependent MHD equations conserve the symmetry relations in time. For example, applying the relations, the divergence term of the first MHD Eq. (A1) is axisymmetric with respect to the xy-plane as a function of $z$ and axisymmetric with respect to the xz-plane as a function of $y$. Thus, Eq. (A1) requires the density to be axisymmetric in time. Because the initial situation (solar wind and Earth's magnetic field) satisfies the symmetry relations and these relations are conserved in time, the solution in its final stationary state needs to satisfy the symmetries, too.

\section{Appendix B}

\section{Second-order equations}

The ansatz (11)-(18) is expanded up to the third order. Substituting this ansatz in the MHD equations, we derived the zeroth- and first-order equations as presented in Sect. 2.1. Analogously, the second-order equations are obtained by equating coefficients of the second order:

$$
\begin{gathered}
\left(\rho_{0} u_{\mathrm{x} 20}+\rho_{20} u_{\mathrm{x} 0}\right)^{\prime}+\rho_{0}\left(3 u_{\mathrm{y} 30}+u_{\mathrm{z} 21}\right) \\
+\rho_{20}\left(3 u_{\mathrm{y} 10}+u_{\mathrm{z} 01}\right)-2 \tilde{c}_{\mathrm{y}}\left(\rho_{0} u_{\mathrm{y} 10}\right)^{\prime} \\
-\frac{\Delta c_{\mathrm{y}}}{x_{\mathrm{MS}}}\left(\rho_{0} u_{\mathrm{x} 0}\right)^{\prime}=0 \\
\left(\rho_{0} u_{\mathrm{x} 02}+\rho_{02} u_{\mathrm{x} 0}\right)^{\prime}+\rho_{0}\left(u_{\mathrm{y} 12}+3 u_{\mathrm{z} 03}\right) \\
+\rho_{02}\left(u_{\mathrm{y} 10}+3 u_{\mathrm{z} 01}\right)-2 \tilde{c}_{\mathrm{z}}\left(\rho_{0} u_{\mathrm{z} 01}\right)^{\prime} \\
-\frac{\Delta c_{\mathrm{z}}}{x_{\mathrm{MS}}}\left(\rho_{0} u_{\mathrm{x} 0}\right)^{\prime}=0 \\
\left(B_{\mathrm{x} 01} u_{\mathrm{y} 10}-B_{\mathrm{y} 11} u_{\mathrm{x} 0}\right)^{\prime}+2\left(B_{\mathrm{z} 02} u_{\mathrm{y} 10}+B_{\mathrm{z} 0} u_{\mathrm{y} 12}\right) \\
-2 B_{\mathrm{y} 11} u_{\mathrm{z} 01}-2 \tilde{c}_{\mathrm{z}}\left(B_{\mathrm{z} 0} u_{\mathrm{y} 10}\right)^{\prime}=0 \\
-\left(B_{\mathrm{z} 0} u_{\mathrm{x} 20}+B_{\mathrm{z} 20} u_{\mathrm{x} 0}\right)^{\prime}-3\left(B_{\mathrm{z} 20} u_{\mathrm{y} 10}+B_{\mathrm{z} 0} u_{\mathrm{y} 30}\right) \\
+2 \tilde{c}_{\mathrm{y}}\left(B_{\mathrm{z} 0} u_{\mathrm{y} 10}\right)^{\prime}+\frac{\Delta c_{\mathrm{y}}}{x_{\mathrm{MS}}}\left(B_{\mathrm{z} 0} u_{\mathrm{x} 0}\right)^{\prime}=0
\end{gathered}
$$

$p_{\mathrm{SW}}=g_{p} p_{0}(\tilde{x}=0)$,

$u_{\mathrm{SW}}=g_{u} u_{\mathrm{x} 0}(\tilde{x}=0)$

$$
\begin{gathered}
\left(B_{\mathrm{x} 01} u_{\mathrm{z} 01}-B_{\mathrm{z} 0} u_{\mathrm{x} 20}-B_{\mathrm{z} 02} u_{\mathrm{x} 0}\right)^{\prime}-B_{\mathrm{z} 02} u_{\mathrm{y} 10} \\
-B_{\mathrm{z} 0} u_{\mathrm{y} 12}+B_{\mathrm{y} 11} u_{\mathrm{z} 01}+\frac{\Delta c_{\mathrm{z}}}{x_{\mathrm{MS}}}\left(B_{\mathrm{z} 0} u_{\mathrm{x} 0}\right)^{\prime}=0, \\
\rho_{0}\left(u_{\mathrm{x} 0} u_{\mathrm{x} 20}\right)^{\prime}+\rho_{20} u_{\mathrm{x} 0} u_{\mathrm{x} 0}^{\prime}+2 \rho_{0} u_{\mathrm{x} 20} u_{\mathrm{y} 10}+p_{20}^{\prime} \\
+\left(B_{\mathrm{z} 0} B_{\mathrm{z} 20}\right)^{\prime}-B_{\mathrm{x} 21} B_{\mathrm{z} 0}-B_{\mathrm{x} 01} B_{\mathrm{z} 20} \\
-2 \tilde{c}_{\mathrm{y}} \rho_{0} u_{\mathrm{y} 10} u_{\mathrm{x} 0}^{\prime}-\frac{\Delta c_{\mathrm{y}}}{x_{\mathrm{MS}}} B_{\mathrm{x} 01} B_{\mathrm{z} 0}=0, \\
\rho_{0}\left(u_{\mathrm{x} 02} u_{\mathrm{x} 0}\right)^{\prime}+\rho_{02} u_{\mathrm{x} 0} u_{\mathrm{x} 0}^{\prime}+2 \rho_{0} u_{\mathrm{x} 02} u_{z 01}+p_{02}^{\prime} \\
+\left(B_{\mathrm{z} 0} B_{\mathrm{z} 02}\right)^{\prime}-3 B_{\mathrm{x} 03} B_{\mathrm{z} 0}-B_{\mathrm{x} 01} B_{\mathrm{z} 02} \\
-2 \tilde{c}_{\mathrm{z}}\left(\rho_{0} u_{\mathrm{z} 01} u_{\mathrm{x} 0}^{\prime}-B_{\mathrm{z} 0} B_{\mathrm{z} 02}^{\prime}\right)-\frac{\Delta c_{\mathrm{z}}}{x_{\mathrm{MS}}} B_{\mathrm{x} 01} B_{\mathrm{z} 0}=0 .
\end{gathered}
$$

\section{Appendix C}

\section{Solving the Rankine-Hugoniot relations at the bow shock}

The MHD moment values of the shocked solar wind plasma at the bow shock's position at $(\tilde{x}=0)$ are $\rho(\tilde{x}=0, y, z)$, $p(\tilde{x}=0, y, z), \boldsymbol{u}(\tilde{x}=0, y, z)$, and $\boldsymbol{B}(\tilde{x}=0, y, z)$. Via the Rankine-Hugoniot relations (31)-(36), they are related to the solar wind conditions introduced in Sect. 2.1: $\rho_{\mathrm{SW}}, p_{\mathrm{SW}}$, $\boldsymbol{u}_{\mathrm{SW}}=\left(u_{\mathrm{SW}}, 0,0\right)^{T}$, and $\boldsymbol{B}_{\mathrm{SW}}=\left(0,0, B_{\mathrm{SW}}\right)^{T}$. On the stagnation streamline, i.e., $y=z=0$, only zeroth-order coefficients of our ansatz (11)-(18) remain and only a solar wind flow normal to the bow shock surface has to be considered. This simple scenario results in the following solution of the Rankine-Hugoniot relations:

$\rho_{\mathrm{SW}}=g_{\rho} \rho_{0}(\tilde{x}=0)$,

$B_{\mathrm{SW}}=g_{B} B_{\mathrm{z} 0}(\tilde{x}=0)$.

Explicit analytical expressions for $g_{\rho}, g_{p}, g_{u}$, and $g_{B}$ depending on the sonic and Alfvénic Mach number of the solar wind can be found in, e.g., Siscoe (1983). In the limit of high Mach numbers, $g_{\rho}=1 / 4, g_{p} \rightarrow 0, g_{u}=4$, and $g_{B}=1 / 4$.

To determine the boundary values at $\tilde{x}=0$ for the higherorder coefficient functions, we need to consider the situation beside the stagnation streamline. This requires taking the bow shock geometry into account, and, consequently, we introduce curvilinear bow shock coordinates. Using the bow shock parametrization (Eq. 9), the normal vector $\boldsymbol{\xi}_{\text {BS }}$ and two 
linearly independent tangential vectors $\boldsymbol{\tau}_{\mathrm{BS}, 1}$ and $\tilde{\boldsymbol{\tau}}_{\mathrm{BS}, 2}$ with respect to the shock are determined:

$\xi_{\mathrm{BS}}=\frac{1}{n_{\mathrm{yz}}}\left(1,-2 c_{\mathrm{BS}, \mathrm{y}} y,-2 c_{\mathrm{BS}, \mathrm{z}} z\right)^{T}$,

$\boldsymbol{\tau}_{\mathrm{BS}, 1}=\frac{1}{n_{\mathrm{y}}}\left(2 c_{\mathrm{BS}, \mathrm{y}} y, 1,0\right)^{T}$,

$\tilde{\boldsymbol{\tau}}_{\mathrm{BS}, 2}=\frac{1}{n_{\mathrm{z}}}\left(2 c_{\mathrm{BS}, \mathrm{z}} z, 0,1\right)^{T}$,

where $n_{\mathrm{yz}}=\sqrt{1+\sum_{t=y, z} 4 c_{\mathrm{BS}, t}^{2} t^{2}}, \quad n_{\mathrm{y}}=\sqrt{1+4 c_{\mathrm{BS}, \mathrm{y}}^{2} y^{2}}$, and $n_{\mathrm{z}}=\sqrt{1+4 c_{\mathrm{BS}, \mathrm{z}}^{2} z^{2}}$ are normalization functions. It is convenient to orthonormalize the tangential vector $\tilde{\boldsymbol{\tau}}_{\mathrm{BS}, 2}$ with respect to the other coordinate vectors $\xi_{\mathrm{BS}}$ and $\boldsymbol{\tau}_{\mathrm{BS}, 1}$ to simplify the following calculations:

$\boldsymbol{\tau}_{\mathrm{BS}, 2}=\frac{\tilde{\boldsymbol{\tau}}_{\mathrm{BS}, 2}-\left(\boldsymbol{\tau}_{\mathrm{BS}, 1} \cdot \tilde{\boldsymbol{\tau}}_{\mathrm{BS}, 2}\right) \boldsymbol{\tau}_{\mathrm{BS}, 1}}{\left\|\tilde{\boldsymbol{\tau}}_{\mathrm{BS}, 2}-\left(\boldsymbol{\tau}_{\mathrm{BS}, 1} \cdot \tilde{\boldsymbol{\tau}}_{\mathrm{BS}, 2}\right) \boldsymbol{\tau}_{\mathrm{BS}, 1}\right\|}$.

The three coordinate vectors $\boldsymbol{\xi}_{\mathrm{BS}}, \boldsymbol{\tau}_{\mathrm{BS}, 1}$, and $\boldsymbol{\tau}_{\mathrm{BS}, 2}$ form an orthonormal system at the bow shock's surface.

First, we consider the shocked velocity at the bow shock plane, which can be expressed by

$\boldsymbol{u}(\tilde{x}=0, y, z)=u_{\xi, \mathrm{BS}} \boldsymbol{\xi}_{\mathrm{BS}}+\sum_{i=1,2} u_{\tau, \mathrm{BS}, i} \boldsymbol{\tau}_{\mathrm{BS}, i}$.

The coefficient functions $u_{\xi, \mathrm{BS}}, u_{\tau, \mathrm{BS}, 1}$, and $u_{\tau, \mathrm{BS}, 2}$ need to be determined. Consider the xz-plane; parametrization of the bow shock curve by introducing the angle $\alpha(z)$ (see Fig. C1) allows for the representation of the solar wind velocity's and magnetic field's component normal to the bow shock as

$u_{\xi, \mathrm{SW}}=\cos (\alpha(z)) u_{\mathrm{SW}} \approx u_{\mathrm{SW}}$,

$B_{\xi, \mathrm{SW}}=\sin (\alpha(z)) B_{\mathrm{SW}} \approx \alpha(z) B_{\mathrm{SW}}$

and the tangential components as

$u_{\tau, \mathrm{SW}}=\sin (\alpha(z)) u_{\mathrm{SW}} \approx \alpha(z) u_{\mathrm{SW}}$,

$B_{\tau, \mathrm{SW}}=\cos (\alpha(z)) B_{\mathrm{SW}} \approx B_{\mathrm{SW}}$.

Here, Taylor expansion up to the first order in $\alpha$ was used. Apparently, the solar wind's normal velocity and tangential magnetic field remain approximately constant close to the stagnation streamline ( $\operatorname{small} \alpha$ ), whereas the normal magnetic field component and the tangential velocity vanish.

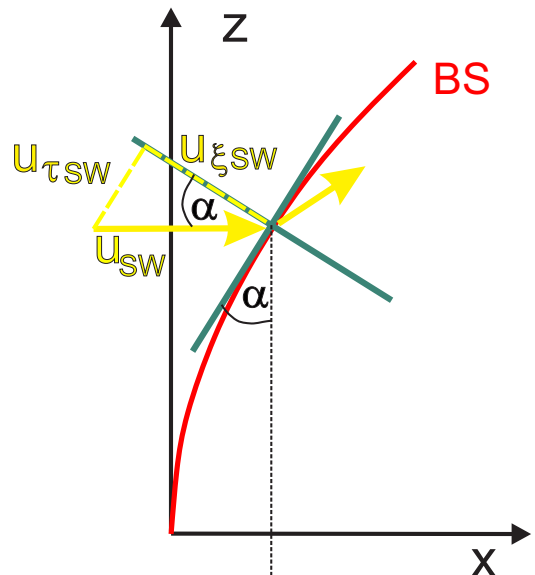

Fig. C1. Projection of the solar wind velocity vector with respect to curvilinear bow shock coordinates.

Note that variations in tangential direction (of the components with respect to the shock's surface), given by derivatives with respect to $\alpha$, require the opposite. As a consequence of the negligible normal magnetic field component, the Rankine-Hugoniot relations require continuity of the tangential velocity components of the solar wind across the bow shock (Siscoe, 1983):

$u_{\tau, \mathrm{BS}, 1}=\boldsymbol{u}_{\mathrm{SW}} \cdot \boldsymbol{\tau}_{\mathrm{BS}, \mathbf{1}}=\frac{2 c_{\mathrm{BS}, \mathrm{y}} u_{\mathrm{SW}} y}{n_{\mathrm{y}}}$,

$u_{\tau, \mathrm{BS}, 2}=\boldsymbol{u}_{\mathrm{SW}} \cdot \boldsymbol{\tau}_{\mathrm{BS}, 2}=\frac{2 c_{\mathrm{BS}, \mathrm{z}} u_{\mathrm{SW}} z}{n_{\mathrm{y}} n_{\mathrm{yz}}}$.

Relation (C3) holds for a normal solar wind velocity with a tangential magnetic field. Because the magnetic field is nearly tangential close to the subsolar point, we extent the scope of this relation to the vicinity of the stagnation streamline using the normal component of the velocities:

$\boldsymbol{u}_{\mathrm{SW}} \cdot \boldsymbol{\xi}_{\mathrm{BS}}=g_{u} \boldsymbol{u}(\tilde{x}=0, y, z) \cdot \boldsymbol{\xi}_{\mathrm{BS}}$.

With the solar wind flowing along the x-axis, Eqs. (C3), (C5), and (C9) yield

$u_{\xi, \mathrm{BS}}=\frac{u_{\mathrm{x} 0}(\tilde{x}=0)}{n_{\mathrm{yz}}}$.

Substituting the coefficients (C14), (C15), and (C17) and the coordinate vectors $\boldsymbol{\xi}_{\mathrm{BS}}, \boldsymbol{\tau}_{\mathrm{BS}, 1}$, and $\boldsymbol{\tau}_{\mathrm{BS}, 2}$ in Eq. (C9), the initial velocity writes

$\boldsymbol{u}(\tilde{x}=0, y, z)=\frac{\Delta u}{n_{\mathrm{yz}}}\left(u_{\mathrm{SW}} \frac{n_{\mathrm{yz}}}{\Delta u}-1,2 c_{\mathrm{BS}, \mathrm{y}} y, 2 c_{\mathrm{BS}, \mathrm{z}} z\right)^{T}$,

where $\Delta u=u_{\mathrm{SW}}-u_{\mathrm{x} 0}(\tilde{x}=0)$. Expanding this relation into a Taylor series with respect to $y$ and $z$ around the stagnation 
streamline and equating coefficients with the ansatz (16)(18) yields values for the coefficient functions that can be expressed as

$u_{l i j}(\tilde{x}=0)=f_{u}(i, j) 2^{i+j} c_{\mathrm{BS}, \mathrm{y}}^{i} c_{\mathrm{BS}, \mathrm{z}}^{j} \Delta u$,

where $l \in\{x, y, z\}$, the indices $i$ and $j$ label the coefficient functions as in the ansatz (16)-(18), and $f_{u}(i, j)$ denotes the sign-function:

$f_{u}(i, j)=\left\{\begin{array}{ll}+1, & i+j \in\{1\} \\ -1, & i+j \in\{2,3\}\end{array}\right.$.

The magnetic field can be treated in a similar manner. Its postshock values are expressed as follows:

$\boldsymbol{B}(\tilde{x}=0, y, z)=B_{\xi, \mathrm{BS}} \boldsymbol{\xi}_{\mathrm{BS}}+\sum_{i=1,2} B_{\tau, \mathrm{BS}, i} \boldsymbol{\tau}_{\mathrm{BS}, i}$.

The normal component of the magnetic field is always continuous through the bow shock, which yields

$B_{\xi, \mathrm{BS}}=\frac{-2 B_{\mathrm{SW}} c_{\mathrm{BS}, \mathrm{z}} z}{n_{\mathrm{yz}}}$.

Similar to the velocity calculation, we extend the validity of relation (C4) to the vicinity of the stagnation streamline:

$\boldsymbol{B}_{\mathrm{SW}} \cdot \boldsymbol{\tau}_{\mathrm{BS}, i}=g_{B} \boldsymbol{B}(\tilde{x}=0, y, z) \cdot \boldsymbol{\tau}_{\mathrm{BS}, i}$,

where $i \in\{1,2\}$. With the solar wind magnetic field along the z-axis, Eqs. (C4), (C6), (C8), and (C21) give one

$B_{\tau, \mathrm{BS}, 1}=0$,

$B_{\tau, \mathrm{BS}, 2}=\frac{B_{\mathrm{z} 0}(0) n_{\mathrm{y}}}{u_{\mathrm{z}}}$.

Finally, the postshock magnetic field is

$$
\begin{gathered}
\boldsymbol{B}(\tilde{x}=0, y, z)=\frac{\Delta B}{n_{\mathrm{yz}}^{2}}\left(2 c_{\mathrm{BS}, \mathrm{z}} z,-4 c_{\mathrm{BS}, \mathrm{y}} c_{\mathrm{BS}, \mathrm{z}} y z,\right. \\
\left.\frac{B_{\mathrm{z} 0}(0)+4 B_{\mathrm{SW}} c_{\mathrm{BS}, \mathrm{y}}^{2} y^{2}+4 B_{\mathrm{SW}} c_{\mathrm{BS}, \mathrm{z}}^{2} z^{2}}{\Delta B}\right)^{T},
\end{gathered}
$$

where $\Delta B=B_{\mathrm{SW}}-B_{\mathrm{Z} 0}(\tilde{x}=0)$. The field boundary values on the stagnation streamline result from the derivatives of Eqs. (13)-(15) at ( $\tilde{x}=0, y=0, z=0)$ and of Eq. (C26):

$B_{l i j}(0)=f_{B}(i, j) 2^{i+j} c_{\mathrm{BS}, \mathrm{y}}^{i} c_{\mathrm{BS}, \mathrm{z}}^{j} \Delta B$,

in which

$f_{B}(i, j)=\left\{\begin{array}{ll}+1, & i+j \in\{1,2\} \\ -1, & i+j \in\{3\}\end{array}\right.$,

where $l \in\{x, y, z\}$, and the indices $i$ and $j$ label the coefficient functions as in the ansatz (13)-(15).

Note that Eqs. (C19) and (C27) satisfy the Frozen-in conservation Eq. (29) as expected. Under the assumptions applied in these calculations, the boundary values for the density and pressure coefficients $\rho_{20}, \rho_{02}, p_{20}$, and $p_{02}$ are zero.

\section{Appendix D}

\section{Zeroth-order approach}

To obtain a first approach to the physics and the corresponding solutions of the MHD equations, the zeroth-order approach with its Eqs. (20)-(23) is solved analytically. The coefficient functions of the first order are set to constant postshock values: $B_{\mathrm{x} 01}=B_{\mathrm{x} 01}(\tilde{x}=0), u_{\mathrm{y} 10}=u_{\mathrm{y} 10}(\tilde{x}=0)$, and $u_{\mathrm{z} 01}=u_{\mathrm{z} 01}(\tilde{x}=0)$. The latter two expressions are called divergence parameters describing the amount of flow diversion in the respective tangential direction. The system is solved for arbitrary solar wind conditions.

\section{D1 General solutions}

For simplification, we neglect magnetic shear, meaning magnetic fields in z-direction only, and consequently $B_{\mathrm{x} 01}(\tilde{x}=$ $0)=0$. The shock front decelerates and compresses the flow; dynamic pressure is converted into gas and magnetic pressure. Most often, in the magnetosheath, the dynamic pressure term in the momentum equation of zeroth order (22) can be neglected (the minor effects of the dynamic pressure are discussed in Sect. 4.1). This yields

$k\left(\rho_{0}^{\gamma}\right)^{\prime}+\frac{1}{\mu_{0}} B_{\mathrm{z} 0} B_{\mathrm{z} 0}^{\prime}=0$,

for which the zeroth-order adiabatic law (Eq. 23) has been used. Integration gives

$k\left(\rho_{0}^{\gamma}\right)+\frac{B_{\mathrm{z} 0}^{2}}{2 \mu_{0}}=k \rho_{0}(0)^{\gamma}+\frac{B_{\mathrm{z} 0}(0)^{2}}{2 \mu_{0}}$,

with the integration constant on the right side to be determined at the bow shock. The zeroth-order continuity Eq. (20) and the zeroth-order Frozen-in theorem (21) can be written as

$u_{\mathrm{x} 0}^{\prime}=-\left(u_{\mathrm{y} 10}+u_{\mathrm{z} 01}\right)+\epsilon$,

$u_{\mathrm{x} 0}^{\prime}=-u_{\mathrm{y} 10}-\delta$,

where $\epsilon=-u_{\mathrm{x} 0} \partial_{\mathrm{x}} \rho_{0} / \rho_{0}$ and $\delta=u_{\mathrm{x} 0} \partial_{\mathrm{x}} B_{\mathrm{z} 0} / B_{\mathrm{z} 0}$. The northward magnetic field and the density are positive functions. Furthermore, $\epsilon$ and $\delta$ are always positive because the magnetic field increases in the magnetosheath, corresponding to a decreasing density as given by Eq. (D1). The positive $\epsilon$ and $\delta$, and Eqs. (D3) and (D4) also provide lower and upper bounds for the velocity decrease:

$-\left(u_{\mathrm{y} 10}+u_{\mathrm{z} 01}\right)<u_{\mathrm{x} 0}^{\prime}<-u_{\mathrm{y} 10}$.

Expanding the velocity in $\tilde{x}$ around the bow shock reads

$u_{\mathrm{x} 0}=u_{\mathrm{x} 0}(0)+\sum_{i=1}^{\infty} a_{i} \tilde{x}^{i}$. 
Note that the argument of the coefficient functions is with respect to $\tilde{x}$, so $u_{\mathrm{x} 0}(0)=u_{\mathrm{x} 0}(\tilde{x}=0)$ and is not explicitly noted further. First, we take only the first order in $\tilde{x}$ into account:

$u_{\mathrm{x} 0}=u_{\mathrm{x} 0}(0)+a \tilde{x}$.

Here $a=a_{1}$ for the expansion coefficient was used. An upper bound for the error of the linear approach can be estimated using Eq. (D5). However, an almost-linear decrease of the velocity along the stagnation streamline is also in accord with global MHD simulation results by, e.g., Wu (1992) or Wang et al. (2004), and also agrees with the observations displayed in Fig. 1. Note that in the hydrodynamic limit the density is constant (incompressible fluid) because of Eq. (D1). This leads to $\epsilon=0$ with the consequence of a linear velocity decrease with $a=-u_{\mathrm{y} 10}-u_{\mathrm{z} 01}$. Substituting the velocity ansatz (D7) in the zeroth-order continuity Eq. (20) and the Frozen-in theorem (21) gives

$\rho_{0}=\rho_{0}(0)\left(\frac{u_{\mathrm{x} 0}}{u_{\mathrm{x} 0}(0)}\right)^{-\frac{u_{\mathrm{y} 10}+u_{\mathrm{z} 01}+a}{a}}$,

$B_{\mathrm{Z} 0}=B_{\mathrm{z} 0}(0)\left(\frac{u_{\mathrm{x} 0}}{u_{\mathrm{x} 0}(0)}\right)^{-\frac{u_{\mathrm{y} 10}+a}{a}}$.

An expression for the expansion parameter $a$ is obtained by substituting the results for $\rho_{0}$ and $B_{\mathrm{z} 0}$ in the simplified momentum equation (D2):

$$
\begin{aligned}
& k\left(\rho_{0}(0)\left(\frac{u_{\mathrm{x} 0}}{u_{\mathrm{x} 0}(0)}\right)^{-\frac{u_{\mathrm{y} 10}+u_{\mathrm{z} 01}+a}{a}}\right)^{\gamma} \\
& +\frac{1}{2 \mu_{0}}\left(B_{\mathrm{z} 0}(0)\left(\frac{u_{\mathrm{x} 0}}{u_{\mathrm{x} 0}(0)}\right)^{-\frac{u_{\mathrm{y} 10}+a}{a}}\right)^{2} \\
& =k \rho_{0}(0)^{\gamma}+\frac{B_{\mathrm{Z} 0}(0)^{2}}{2 \mu_{0}} \text {. }
\end{aligned}
$$

To solve this equation, both sides are expanded into Taylor series around $\tilde{x}=0$. Equating coefficients of the lowest nonvanishing order yields

$a=-u_{\mathrm{y} 10}-u_{\mathrm{z} 01}\left(1-\frac{1}{1+\frac{\gamma}{2} \frac{p_{0}(0)}{p_{\operatorname{mag}}(0)}}\right)$.

Here $p_{\text {mag }}(0)=B_{\mathrm{z} 0}^{2}(0) / 2 \mu_{0}$ denotes the postshock magnetic pressure and $\gamma=5 / 3$ the ratio of specific heats.

This result is used to obtain an expression for the magnetosheath thickness $x_{\text {MS }}$. The magnetopause is defined by a vanishing flow velocity on the stagnation streamline $\left(u_{\mathrm{x}}\left(x_{\mathrm{MS}}\right)=0\right)$. Equation (D7) thus yields

$x_{\mathrm{MS}}=\frac{u_{\mathrm{x} 0}(0)}{a}$.
After substitution of Eq. (D11), we obtain

$x_{\mathrm{MS}}=\frac{-u_{\mathrm{x} 0}(0)}{u_{\mathrm{y} 10}+m_{\mathrm{BS}} u_{\mathrm{z} 01}}$,

with

$m_{\mathrm{BS}}=1-\frac{1}{1+\frac{\gamma}{2} \frac{p_{0}(0)}{p_{\operatorname{mag}}(0)}}$.

The parameter $m_{\mathrm{BS}}$ is a measure for the solar wind magnetization. For the unmagnetized solar wind, we obtain $m_{\mathrm{BS}}=1$. Note that the magnetosheath expression results from ideal MHD equations, neglecting magnetic shear $\left(B_{\mathrm{x} 01}(0)=0\right)$ and the dynamic pressure terms, and using a series ansatz for the velocity $\left(\boldsymbol{u}=\left(u_{\mathrm{x} 0}(0)+a \tilde{x}, u_{\mathrm{y} 01}(0), u_{\mathrm{z} 10}(0)\right)^{T}\right)$.

Higher-order contributions of the velocity to the magnetosheath thickness can also be obtained. For a second-order ansatz for the velocity in $\tilde{x}$-direction, similar calculations yield the second-order expansion coefficient:

$a_{2}=\frac{2 \gamma^{2} p_{\mathrm{mag}}(0) p_{0}(0)\left(p_{\mathrm{mag}}(0)+p_{0}(0)\right) u_{\mathrm{z} 01}^{2}}{\left(2 p_{\mathrm{mag}}(0)+\gamma p_{0}(0)\right)^{3} u_{\mathrm{x} 0}(0)}$.

In the limit $p_{0}(0)>>p_{\text {mag }}(0)$ the expression is approximated by:

$a_{2} \approx 2 \gamma \frac{p_{\mathrm{mag}}(0)}{p_{0}(0)} \frac{u_{\mathrm{z} 01}^{2}}{u_{\mathrm{x} 0}(0)}$.

Consistently, $a_{2}$ vanishes in the limit of hydrodynamics (i.e., $\left.p_{\text {mag }}(0)=0\right)$ and a linear velocity decrease remains.

\section{D2 Boundary conditions}

The divergence parameters are set to their bow shock values, which are given by Eq. (C19):

$u_{\mathrm{y} 10}=2 c_{\mathrm{BS}, \mathrm{y}} \Delta u$,

$u_{\mathrm{z} 01}=2 c_{\mathrm{BS}, \mathrm{z}} \Delta u$.

The geometry parameters $c_{\mathrm{BS}, \mathrm{y}}$ and $c_{\mathrm{BS}, \mathrm{z}}$ need to be determined.

The dynamic pressure of the solar wind is completely converted into gas and magnetic pressure in the magnetosheath. However, pressure is not a conserved quantity and, thus, the solar wind pressure differs by a factor $K$ from the magnetopause pressure on the stagnation streamline:

$K \rho_{\mathrm{SW}} u_{\mathrm{SW}}^{2}=p_{\mathrm{tot}, \mathrm{MP}}$

(D19)

where the total magnetopause pressure $p_{\text {tot,MP }}$ is given by Eq. (48). Here $K \approx 0.89$ holds for a broad range of solar wind conditions (Kivelson and Russell, 1995). 
The pressure balance equation above is valid at the stagnation point $\left(\tilde{x}=x_{\mathrm{MS}}, y=0, z=0\right)$ only. In its vicinity, only the velocity component normal to the local magnetopause is important. The dynamic pressure with respect to the magnetopause normal (Eq. 41) thus reads

$p_{\mathrm{dyn}, \xi}=\rho_{\mathrm{SW}} u_{\mathrm{SW}, \xi}^{2}=\rho \frac{u_{\mathrm{SW}}^{2}}{n_{\mathrm{MP}}^{2}}$.

This expression holds for both the $y$ - and z-directions. Herewith, the pressure equilibrium writes

$K p_{\text {dyn }, \xi}=p_{\text {tot,MP }}$,

where Eq. (48) was used and $f=2.44$. Note that by setting $K=1$ the pressure relation becomes equivalent to the one used by Mead and Beard (1964).

Using the magnetopause parametrization (10), the Earth's dipole field (Eqs. 6-8), the magnetopause normal vector (Eq. 41), and the magnetospheric pressure (Eq. 48) in the xy-plane yield

$$
p_{\text {tot,MP,xy }}=\frac{f^{2}}{2 \mu_{0}} \frac{M^{2}}{\left(\left(\Delta x_{\mathrm{MP}}-c_{\mathrm{MP}, \mathrm{y}} y^{2}\right)^{2}+y^{2}\right)^{3}} .
$$

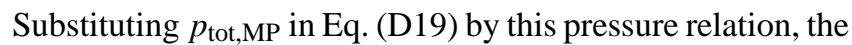
magnetopause distance $\Delta x_{\mathrm{MP}}$ at the stagnation point $(y=0)$ is obtained, given by the well-know expression (e.g., $\mathrm{Pu}-$ dovkin et al., 1998)

$$
\Delta x_{\mathrm{MP}}=\left(\frac{f^{2} M^{2}}{2 \mu_{0} K \rho_{\mathrm{SW}} u_{\mathrm{SW}}^{2}}\right)^{\frac{1}{6}} .
$$

The geomagnetic dipole moment is given by $M=8 \times$ $10^{15} \mathrm{Tm}^{3}$. The general pressure balance (D21) along with Eqs. (D20) and (D22) is solved using Taylor expansion with respect to $y$ around the stagnation point up to the second order. Equating coefficients allows for determination of the magnetopause curvature:

$c_{\mathrm{MP}, \mathrm{y}}=\frac{-3+\sqrt{21}}{4 \Delta x_{\mathrm{MP}}} \approx \frac{0.4}{\Delta x_{\mathrm{MP}}}$.

This expression now enables us to estimate the parabolic coefficient $c_{\mathrm{BS}, \mathrm{y}}$, which we need to know for an estimate of the postshock divergence. We assume the same functional expression for the curvature of the bow shock as derived for the magnetopause above:

$c_{\mathrm{BS}, \mathrm{y}}=\frac{0.4}{\Delta x_{\mathrm{BS}}}=\frac{0.4}{\Delta x_{\mathrm{MP}}+x_{\mathrm{MS}}}$.

The divergence parameter $u_{\mathrm{y} 10}$ is now calculated with Eq. (D17):

$u_{\mathrm{y} 10}=0.8 \frac{u_{\mathrm{SW}}-u_{\mathrm{x} 0}(0)}{\Delta x_{\mathrm{MP}}+x_{\mathrm{MS}}}$, where $\Delta u=u_{\mathrm{SW}}-u_{\mathrm{x} 0}(0)$ was used, as defined above.

The velocity divergence within the xz-plane remains to be determined. Using Eq. (48) to describe the magnetospheric pressure within the xz-plane leads to

$p_{\text {tot,MP, }, \mathrm{xz}}=\frac{\left(2 z^{2}-\Delta x^{2}-6 c_{\mathrm{MP}, \mathrm{z}} \Delta x z^{2}\right)^{2}}{\left(1+4 c_{\mathrm{MP}, \mathrm{z}}^{2} z^{2}\right)\left(\Delta x^{2}+z^{2}\right)^{5}} \frac{f^{2} M^{2}}{2 \mu_{0}}$,

which yields

$c_{\mathrm{MP}, \mathrm{z}}=\frac{1}{2 \Delta x_{\mathrm{MP}}}$.

Assuming, again, the same functional expression for the bow shock curvature gives

$c_{\mathrm{BS}, \mathrm{z}}=\frac{1}{2 \Delta x_{\mathrm{BS}}}=\frac{1}{2\left(\Delta x_{\mathrm{MP}}+x_{\mathrm{MS}}\right)}$,

and using Eq. (D18) the divergence parameter $u_{\mathrm{z} 01}$ reads

$u_{\mathrm{z} 01}=\frac{u_{\mathrm{SW}}-u_{\mathrm{x} 0}(0)}{\Delta x_{\mathrm{MP}}+x_{\mathrm{MS}}}$.

Altogether, after some tedious calculations, Eqs. (D26) and (D30) provide suitable estimates for flow divergence just after the bow shock. The numerator is determined by the velocity jump across the bow shock, and the denominator by the bow shock's distance to the Earth's center. The ratio of both divergence parameters is

$u_{\mathrm{y} 10}=\frac{4}{5} u_{\mathrm{z} 01}$.

This equation describes the asymmetry of the divergence in $y$ - and z-direction in the approach discussed.

The expressions for the divergence still depend on the magnetosheath thickness. With Eqs. (C3), (D13), (D26), and (D30) we get

$x_{\mathrm{MS}}=\frac{\Delta x_{\mathrm{MP}}}{\left(\frac{4}{5}+m_{\mathrm{BS}}\right)\left(g_{u}-1\right)-1}$.

This relation for the magnetosheath thickness depends on the solar wind conditions only. The velocity jump at the bow shock $g_{u}$ (Eq. C3), the magnetopause distance form the Earth's center $\Delta x_{\mathrm{MP}}$ (Eq. D23), and the solar wind magnetization $m_{\mathrm{BS}}$ (Eq. D14) are explicit functions of the solar wind parameters. Equations $(\mathrm{C} 1)-(\mathrm{C} 4)$ are used to refer the bow shock conditions $\rho_{0}(0), p_{0}(0), B_{\mathrm{z} 0}(0)$, and $u_{\mathrm{x} 0}(0)$ back to solar wind conditions. Hence, Eqs. (D26) and (D30) can also be written as explicit functions of these solar wind parameters.

\section{D3 Application}

The flow velocity, the density, and the magnetic field are given by Eqs. (D7), (D8), and (D9), with the expansion coefficient given by Eq. (D11). The postshock boundary conditions $u_{\mathrm{x} 0}(0), \rho_{0}(0), B_{\mathrm{z} 0}(0)$, and $p_{0}(0)$ are related to the 
solar wind conditions via Eqs. (C1-C4). Furthermore, the divergence parameters $u_{\mathrm{y} 10}$ and $u_{\mathrm{z} 01}$ were set constant to their postshock boundary values given by the relations (D26) and (D30). This requires the magnetopause distance $\Delta x_{\mathrm{MP}}$ determined by Eq. (D23) and the magnetosheath thickness by Eq. (D32).

Acknowledgements. This work was financially supported by the German Ministerium für Wirtschaft und Technologie and the Deutsches Zentrum für Luft- und Raumfahrt under contracts 50OC1001, 50OC1102, and 50QP1001. We acknowledge NASA contract NAS5-02099 and V. Angelopoulos for use of data from the THEMIS Mission. Specifically: C. W. Carlson and J. P. McFadden for use of ESA data.

Topical Editor L. Blomberg thanks S. Fujita and one anonymous referee for their help in evaluating this paper.

\section{References}

Angelopoulos, V.: The THEMIS mission, Space Sci. Rev., 141, 534, doi:10.1007/s11214-008-9336-1, 2008.

Auster, H. U., Glassmeier, K.-H., Magnes, W., Aydogar, O., Baumjohann, W., D. Constantinescu, D., Fischer, D., Fornacon, K. H. , Georgescu, E., Harvey, P., Hillenmaier, O., Kroth, R., Ludlam, M., Narita, Y., Nakamura, R., Okrafka, K., Plaschke, F., Richter, I., Schwarzl, H., Stoll, B., Valavanoglou, A., and Wiedemann, M.: The THEMIS fluxgate magnetometer, Space Sci. Rev., 141, 235-264, doi:10.1007/s11214-008-9365-9, 2008.

Auster, H. U., Richter, I., Glassmeier, K.-H., Berghofer, G., Carr, C. M., and Motschmann, U.: Magnetic Field Investigations during ROSETTA's 2867 Steins Flyby, Planet. Space Sci. 58, 1124 1128, 2010.

Bennett, L., Kivelson, M. G. , Khurana, K. K., Frank, L. A., and Paterson, W. R.: A model of the Earth's distant bow shock, J. Geophys. Res., 102, 26927-26941, doi:10.1029/97JA01906, 1997.

Biernat, H. K., Erkaev, N. V., and Farrugia, C. J.: Aspects of MHD flow about Venus, J. Geophys. Res., 104, 12617-12626, doi:10.1029/1999JA900032, 1999.

Chapman, S. and Ferraro, V. C.: A new theory of magnetic storms, Nature, 126, 129-130, doi:10.1038/126129a0, 1930.

Crooker, N. U., Eastman, T. E., and Stiles, G. S.: Observations of plasma depletion in the magnetosheath at the dayside magnetopause, J. Geophys. Res., 84, 869-874, doi:10.1029/JA084iA03p00869, 1979.

Erkaev, N. V., Farrugia, C. J., and Biernat, H. K.: Effects on the Jovian magnetosheath arising from solar wind flow around nonaxisymmetric bodies, J. Geophys. Res., 101, 10665-10672, 1996.

Erkaev, N. V.: Results of the investigation of MHD flow around the magnetosphere, Geomagn. Aeron., 28, 455-464, 1988.

Erkaev, N. V., Biernat, H. K., and Farrugia, C. J.: Ideal magnetohydrodynamic flow around a blunt body under anisotropic pressure, Phys. Plasmas, 7, 3413-3420, 2000.

Erkaev, N. V., Farrugia, C. J., and Biernat, H. K.: The role of magnetic barrier in the Solar-wind-magnetosphere interaction, Planet. Space Sci. 51, 745-755, doi:10.1016/S00320633(03)00111-9, 2003.

Farris, M. H. and Russell, C. T.: Determining the standoff distance of the bow shock: Mach number dependence and use of models,
J. Geophys. Res., 99, 17681-17689, doi:10.1029/94JA01020, 1994.

Farrugia, C. J., Erkaev, N. V., Biernat, H. K., Lawrence, G. R., and Elphic, R. C.: Plasma depletion layer model for low Alfven Mach number: Comparison with ISEE observations, J. Geophys. Res., 102, 11315-11324, doi:10.1029/97JA00410, 1997.

Glassmeier, K.-H., Boehnhardt, H. , Koschny, D., Kuehrt, E., and Richter, I.: The ROSETTA Mission: Flying towards the Origin of the Solar System, Space Sci. Rev., 128, 1-21, doi:10.1007/s11214-006-9140-8, 2007.

Glassmeier, K.-H., Auster, H.-U., Constantinescu, D., Fornacon, K.H., Narita, Y., Plaschke, F., Angelopoulos, V., Georgescu, E., Baumjohann, W., Magnes, W., Nakamura, R., Carlson, C. W., Frey, S., McFadden, J. P., Phan, T., Mann, I., Rae, I. J., and Vogt, J.: Magnetospheric quasi-static response to the dynamic magnetosheath: A THEMIS case study, Geophys. Res. Lett., 35, L17S01, doi:10.1029/2008GL033469, 2008.

Kivelson, M. G. and Russell, C. T.: Introduction to space physics, Cambridge University Press, 1995.

Lees, L.: Interaction between the solar plasma wind and the geomagnetic cavity, AIAA J., 2, 1576-1582, 1964.

McFadden, J. P., Carlson, C. W., Larson, D., Ludlam, M., Abiad, R., Elliott, B., Turin, P., Marckwordt, M., and Angelopoulos, V.: The THEMIS ESA plasma instrument and in-flight calibration, Space Sci. Rev., 141, 277-302, doi:10.1007/s11214-008-9440-2, 2008.

Mead, G. D. and Beard, D. B.: Shape of the geomagnetic field solar wind boundary, J. Geophys. Res., 69, 1169-1179, 1964.

Ogino, T.: Two-dimensional MHD code, in: Computer Space Plasma Physics: Simulations and Software, H. Matsumoto and Y. Omura, Terra Scientific Publishing Company, Tokyo, 161-207, 1993.

Ogino, T., Walker, R. J., and Maha, A. A.: A global magnetohydrodynamic simulation of the magnetosheath and magnetosphere when the magnetic interplanetary field is northward, IEEE T. Plasma Sci., 20, 817-828, 1992.

Paschmann, G., Baumjohann, W., Sckopke, N., and Lühr, H.: Structure of the dayside magnetopause for low magnetic shear, J. Geophys. Res., 98, 13409-13422, doi:10.1029/93JA00646, 1993.

Phan, T.-D., Paschmann, G., Baumjohann, W., Sckopke, N., and Lühr, H.: The magnetosheath region adjacent to the dayside magnetopause: AMPTE/IRM observations, J. Geophys. Res., 99, 121-140, doi:10.1029/93JA02444, 1994.

Petrinec, S. M.: The location of the Earth's bow shock, Planet. Space Sci., 50, 541-547, 2002.

Petrinec, S. M. and Russell, C. T.: Hydrodynamics and MHD equations across the bow shock and along the surfaces of planetary obstacles, Space Sci. Rev., 79, 757-791, 1997.

Plaschke, F., Glassmeier, K.-H., Auster, H. U., Constantinescu, O. D., Magnes, W., Angelopoulos, V., Sibeck, D. G., and McFadden, J. P.: Standing Alfven waves at the magnetopause, Geophys. Res. Lett., 36, L02104, doi:10.1029/2008GL036411, 2009.

Pudovkin, M. I. and Semenov, V. S.: Peculiarities of the MHD-flow by the magnetopause and generation of electric field in the magnetosphere, Ann. Geophys., 33, 423-427, 1977a.

Pudovkin, M. I. and Semenov, V. S.: Stationary frozen-in coordinate system, Ann. Geophys., 33, 429-433, 1977b.

Pudovkin, M. I., Besser, B. P., and Zaitseva, S. A.: Magnetopause stand-off distance in dependence on the magnetosheath 
and solar wind parameters, Ann. Geophys., 16, 388-396, doi:10.1007/s00585-998-0388-z, 1998.

Shue, J. H., Song, P., Russel, C. T., Steinberg, J. T., Chao, J. K., Zastenker, G., Vaisberg, O. L., Kokubun, S., Singer, H. J., Detman, T. R., and Kawano, H.: Magnetopause location under extreme solar wind conditions, J. Geophys. Res., 103, 1769117700, doi:10.1029/98JA01103, 1998.

Siscoe, G. L.: Solar System Magnetohydrodynamics, in: Solarterrestrial physics: Principles and theoretical foundations: based on the proceedings of the Theory Institute held at Boston College, 9-26 August 1982, edited by: Carovillano, R. L. and Forbes, J. M., D. Reidel Publishing Co., 11-100, 1983.

Siscoe, G. L., Crooker, N. U., Erickson, G. M., Sonnerup, B. U. O., Maynard, N. C., Schoendorf, J. A., Siebert, K. D.,Weimer, D. R., White, W. W., and Wilson, G. R.: MHD properties of magnetosheath flow, Planet. Space Sci., 50, 461-471, 2002.

Spreiter, J. R., Summers, A. L., and Alksne, A. Y.: Hydromagnetic flow around the magnetosphere, Planet. Space Sci., 14, 223-253, 1966.

Toth, G.: The $\nabla \cdot \boldsymbol{B}$ constraint in shock-capturing Magnetohydrodynamics Codes, J. Comput. Phys., 161, 605-652, 2000.
Wang, Y. L., Raeder, J., and Russell, C. T.: Plasma depletion layer: Magnetosheath flow structure and forces, Ann. Geophys., 22, 1001-1017, doi:10.5194/angeo-22-1001-2004, 2004.

Wu, C. C.: MHD modeling of the Earth's magnetosphere, in: Computer Simulation of Space Plasma, H. Matsumoto and T. Sato, Terra Scientific Puplishing Company, Tokyo, 155-177, 1984.

Wu, C. C.: MHD flow past an obstacle: Large-scale flow in the magnetosheath, Geophys. Res. Lett., 19, 87-90, doi:10.1029/91GL03007, 1992.

Wu, C. C., Walker, R., and Dawson, J. M.: A three-dimensional MHD model of the Earth's magnetosphere, Geophys. Res. Lett., 8, 523-526, doi:10.1029/GL008i005p00523, 1981.

Zhang, H., Zong, Q.-G., Sibeck, D. G., Fritz, T. A., McFadden, J. P., Glassmeier, K.-H., and Larson, D.: Dynamic motion of the bow shock and the magnetopause observed by THEMIS spacecraft, J. Geophys. Res., 114, A00C12, doi:10.1029/2008JA013488, 2009.

Zwan, B. J. and Wolf, R. A.: Depletion of solar wind plasma near a planetary boundary, J. Geophys. Res., 81, 1636-1648, doi:10.1029/JA081i010p01636, 1976. 